\title{
Measurement of the absolute branching fraction of inclusive semielectronic $D_{s}^{+}$decays
}

M. Ablikim, ${ }^{1}$ M. N. Achasov, ${ }^{10, b}$ P. Adlarson,${ }^{67}$ S. Ahmed ${ }^{15}$ M. Albrecht,${ }^{4}$ R. Aliberti, ${ }^{28}$ A. Amoroso, ${ }^{66 a, 66 c}$ M. R. An,${ }^{32}$ Q. An ${ }^{63,49}$ X. H. Bai,${ }^{57}$ Y. Bai, ${ }^{48}$ O. Bakina, ${ }^{29}$ R. Baldini Ferroli, ${ }^{23 a}$ I. Balossino, ${ }^{24 a}$ Y. Ban, ${ }^{38, h}$ K. Begzsuren, ${ }^{26}$ N. Berger ${ }^{28}$ M. Bertani, ${ }^{23 a}$ D. Bettoni, ${ }^{24 a}$ F. Bianchi, ${ }^{66 a, 66 c}$ J. Bloms, ${ }^{60}$ A. Bortone, ${ }^{66 a, 66 c}$ I. Boyko, ${ }^{29}$ R. A. Briere, ${ }^{5}$ H. Cai ${ }^{68}$ X. Cai ${ }^{1,49}$ A. Calcaterra, ${ }^{23 a}$ G. F. Cao, ${ }^{1,54}$ N. Cao, ${ }^{1,54}$ S. A. Cetin, ${ }^{53 a}$ J. F. Chang, ${ }^{1,49}$ W. L. Chang, ${ }^{1,54}$ G. Chelkov, ${ }^{29, a}$ D. Y. Chen, ${ }^{6}$ G. Chen, ${ }^{1}$ H. S. Chen, ${ }^{1,54}$ M. L. Chen, ${ }^{1,49}$ S. J. Chen ${ }^{35}$ X. R. Chen, ${ }^{25}$ Y. B. Chen, ${ }^{1,49}$ Z. J. Chen, ${ }^{20, i}$ W. S. Cheng, ${ }^{66 c}$ G. Cibinetto, ${ }^{24 a}$ F. Cossio, ${ }^{66 c}$ X. F. Cui,${ }^{36}$ H. L. Dai,${ }^{1,49}$ X. C. Dai,${ }^{1,54}$ A. Dbeyssi, ${ }^{15}$ R. E. de Boer, ${ }^{4}$ D. Dedovich, ${ }^{29}$ Z. Y. Deng, ${ }^{1}$ A. Denig, ${ }^{28}$ I. Denysenko,${ }^{29}$ M. Destefanis, ${ }^{66 a, 66 c}$ F. De Mori, ${ }^{66 a, 66 c}$ Y. Ding,${ }^{33}$ C. Dong, ${ }^{36}$ J. Dong, ${ }^{1,49}$ L. Y. Dong, ${ }^{1,54}$ M. Y. Dong, ${ }^{1,49,54}$ X. Dong, ${ }^{68}$ S. X. Du, ${ }^{71}$ Y. L. Fan, ${ }^{68}$ J. Fang, ${ }^{1,49}$ S. S. Fang, ${ }^{1,54}$ Y. Fang, ${ }^{1}$ R. Farinelli, ${ }^{24 a}$ L. Fava ${ }^{66 b, 66 c}$ F. Feldbauer, ${ }^{4}$ G. Felici, ${ }^{23 a}$ C. Q. Feng, ${ }^{63,49}$ J. H. Feng, ${ }^{50}$ M. Fritsch, ${ }^{4}$ C. D. Fu, ${ }^{1}$ Y. Gao, ${ }^{38, h}$ Y. Gao, ${ }^{64}$ Y. Gao ${ }^{63,49}$ Y. G. Gao, ${ }^{6}$ I. Garzia, ${ }^{24 a, 24 b}$ P. T. Ge,${ }^{68}$ C. Geng,${ }^{50}$ E. M. Gersabeck,${ }^{58}$ A. Gilman $\odot,{ }^{61}$ K. Goetzen, ${ }^{11}$ L. Gong, ${ }^{33}$ W. X. Gong, ${ }^{1,49}$ W. Gradl, ${ }^{28}$ M. Greco, ${ }^{66 a, 66 c}$ L. M. Gu, ${ }^{35}$ M. H. Gu, ${ }^{1,49}$ Y. T. Gu, ${ }^{13}$ C. Y. Guan, ${ }^{1,54}$ A. Q. Guo, ${ }^{22}$ L. B. Guo, ${ }^{34}$ R. P. Guo, ${ }^{40}$ Y. P. Guo, ${ }^{9, \mathrm{f}}$ A. Guskov, ${ }^{29, \mathrm{a}}$ T. T. Han ${ }^{41}$ W. Y. Han ${ }^{32}$ X. Q. Hao,${ }^{16}$ F. A. Harris,${ }^{56}$ K. L. He,${ }^{1,54}$ F. H. Heinsius, ${ }^{4}$ C. H. Heinz ${ }^{28}$ T. Held, ${ }^{4}$ Y. K. Heng, ${ }^{1,49,54}$ C. Herold, ${ }^{51}$ M. Himmelreich, ${ }^{11, d}$ T. Holtmann, ${ }^{4}$ G. Y. Hou, ${ }^{1,54}$ Y. R. Hou, ${ }^{54}$ Z. L. Hou ${ }^{1}{ }^{\text {H. M. Hu }}{ }^{1,54}$ J. F. Hu,${ }^{4, j}$ T. Hu,${ }^{1,49,54}$ Y. Hu, ${ }^{1}$ G. S. Huang ${ }^{63,49}$ L. Q. Huang ${ }^{64}$ X. T. Huang, ${ }^{41}$ Y. P. Huang, ${ }^{1}$ Z. Huang, ${ }^{38, h}$ T. Hussain, ${ }^{65}$ N. Hüsken, ${ }^{22,28}$ W. Ikegami Andersson, ${ }^{67}$ W. Imoehl, ${ }^{22}$ M. Irshad,${ }^{63,49}$ S. Jaeger, ${ }^{4}$ S. Janchiv, ${ }^{26}$ Q. Ji, ${ }^{1}$ Q. P. Ji, ${ }^{16}$ X. B. Ji, ${ }^{1,54}$ X. L. Ji, ${ }^{1,49}$ Y. Y. Ji ${ }^{41}$ H. B. Jiang, ${ }^{41}$ X. S. Jiang, ${ }^{1,49,54}$ J. B. Jiao, ${ }^{41}$ Z. Jiao, ${ }^{18}$ S. Jin,${ }^{35}$ Y. Jin,${ }^{57}$

M. Q. Jing, ${ }^{1,54}$ T. Johansson, ${ }^{67}$ N. Kalantar-Nayestanaki, ${ }^{55}$ X. S. Kang, ${ }^{33}$ R. Kappert, ${ }^{55}$ M. Kavatsyuk, ${ }^{55}$ B. C. Ke, ${ }^{43,1}$ I. K. Keshk, ${ }^{4}$ A. Khoukaz ${ }^{60}$ P. Kiese,${ }^{28}$ R. Kiuchi, ${ }^{1}$ R. Kliemt,${ }^{11}$ L. Koch, ${ }^{30}$ O. B. Kolcu, ${ }^{53 a, m}$ B. Kopf,${ }^{4}$ M. Kuemmel, ${ }^{4}$ M. Kuessner, ${ }^{4}$ A. Kupsc, ${ }^{67}$ M. G. Kurth, ${ }^{1,54}$ W. Kühn, ${ }^{30}$ J. J. Lane, ${ }^{58}$ J. S. Lange,${ }^{30}$ P. Larin, ${ }^{15}$ A. Lavania, ${ }^{21}$ L. Lavezzi ${ }^{66 a, 66 c}$

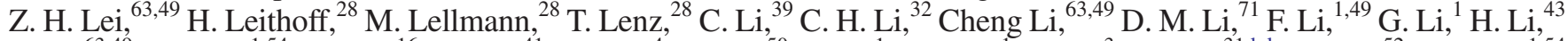
H. Li,${ }^{63,49}$ H. B. Li, ${ }^{1,54}$ H. J. Li, ${ }^{16}$ J. L. Li ${ }^{41}{ }^{11}$ J. Q. Li, ${ }^{4}$ J. S. Li ${ }^{50}{ }^{5 e ~ L i},{ }^{1}$ L. K. Li ${ }^{1}{ }^{1}$ Lei Li, ${ }^{3}$ P. R. Li,${ }^{31, k, 1}$ S. Y. Li, ${ }^{52}$ W. D. Li,${ }^{1,54}$ W. G. Li,${ }^{1}$ X. H. Li,${ }^{63,49}$ X. L. Li ${ }^{41}$ Xiaoyu Li, ${ }^{1,54}$ Z. Y. Li, ${ }^{50}$ H. Liang, ${ }^{6,49}$ H. Liang, ${ }^{1,54}$ H. Liang, ${ }^{27}$ Y. F. Liang, ${ }^{45}$

Y. T. Liang, ${ }^{25}$ G. R. Liao, ${ }^{12}$ L. Z. Liao, ${ }^{1,54}$ J. Libby, ${ }^{21}$ C. X. Lin,${ }^{50}$ B. J. Liu, ${ }^{1}$ C. X. Liu, ${ }^{1}$ D. Liu,${ }^{15,63}$ F. H. Liu, ${ }^{44}$ Fang Liu, ${ }^{1}$ Feng Liu, ${ }^{6}$ H. B. Liu, ${ }^{13}$ H. M. Liu, ${ }^{1,54}$ Huanhuan Liu, ${ }^{1}$ Huihui Liu, ${ }^{17}$ J. B. Liu, ${ }^{63,49}$ J. L. Liu, ${ }^{64}$ J. Y. Liu, ${ }^{1,54}$ K. Liu, ${ }^{1}$

K. Y. Liu, ${ }^{33}$ L. Liu, ${ }^{63,49}$ M. H. Liu, ${ }^{9,}{ }^{7}$ P. L. Liu, ${ }^{1}$ Q. Liu, ${ }^{54}$ Q. Liu, ${ }^{68}$ S. B. Liu, ${ }^{63,49}$ Shuai Liu, ${ }^{46}$ T. Liu, ${ }^{1,54}$ W. M. Liu, ${ }^{63,49}$ X. Liu ${ }^{31, k, 1}$ Y. Liu, ${ }^{31, k, 1}$ Y. B. Liu, ${ }^{36}$ Z. A. Liu, ${ }^{1,49,54}$ Z. Q. Liu, ${ }^{41}$ X. C. Lou, ${ }^{1,49,54}$ F. X. Lu, ${ }^{50}$ H. J. Lu, ${ }^{18}$ J. D. Lu, ${ }^{1,54}$

J. G. Lu, ${ }^{1,49}$ X. L. Lu, ${ }^{1}$ Y. Lu, ${ }^{1}$ Y. P. Lu, ${ }^{1,49}$ C. L. Luo, ${ }^{34}$ M. X. Luo, ${ }^{70}$ P. W. Luo, ${ }^{50}$ T. Luo,,${ }^{9,}$ X. L. Luo, ${ }^{1,49}$ X. R. Lyu ${ }^{54}$ F. C. Ma ${ }^{33}$ H. L. Ma, ${ }^{1}$ L. L. Ma ${ }^{41}$ M. M. Ma ${ }^{1,54}$ Q. M. Ma, ${ }^{1}$ R. Q. Ma,${ }^{1,54}$ R. T. Ma, ${ }^{54}$ X. X. Ma,${ }^{1,54}$ X. Y. Ma, ${ }^{1,49}$ F. E. Maas, ${ }^{15}$ M. Maggiora, ${ }^{66 a, 66 c}$ S. Maldaner, ${ }^{4}$ S. Malde ${ }^{61}$ Q. A. Malik, ${ }^{65}$ A. Mangoni, ${ }^{23 b}$ Y. J. Mao, ${ }^{38, h}$ Z. P. Mao, ${ }^{1}$ S. Marcello, ${ }^{66 a, 66 c}$ Z. X. Meng, ${ }^{57}$ J. G. Messchendorp ${ }^{55}$ G. Mezzadri, ${ }^{24 a}$ T. J. Min, ${ }^{35}$ R. E. Mitchell, ${ }^{22}$ X. H. Mo, ${ }^{1,49,54}$ Y. J. Mo, ${ }^{6}$ N. Yu. Muchnoi ${ }^{10, b}$ H. Muramatsu ${ }^{59}$ S. Nakhoul, ${ }^{11, d}$ Y. Nefedov, ${ }^{29}$ F. Nerling, ${ }^{11, d}$ I. B. Nikolaev, ${ }^{10, b}$ Z. Ning, ${ }^{1,49}$ S. Nisar, ${ }^{8, \mathrm{~g}}$ Q. Ouyang,${ }^{1,49,54}$ S. Pacetti, ${ }^{23 b, 23 \mathrm{c}}$ X. Pan,${ }^{9, \mathrm{f}}$ Y. Pan, ${ }^{58}$ A. Pathak, ${ }^{1}$ A. Pathak ${ }^{27}$ P. Patteri, ${ }^{23 \mathrm{a}}$ M. Pelizaeus ${ }^{4}$ H. P. Peng,${ }^{63,49}$ K. Peters, ${ }^{11, d}$ J. Pettersson, ${ }^{67}$ J. L. Ping, ${ }^{34}$ R. G. Ping, ${ }^{1,54}$ S. Pogodin, ${ }^{29}$ R. Poling ${ }^{59}$ V. Prasad,${ }^{63,49}$ H. Qi,${ }^{63,49}$ H. R. Qi, ${ }^{52}$ K. H. Qi,${ }^{25}$ M. Qi, ${ }^{35}$ T. Y. Qi ${ }^{9}$ S. Qian, ${ }^{1,49}$ W. B. Qian, ${ }^{54}$ Z. Qian, ${ }^{50}$ C. F. Qiao, ${ }^{54}$ L. Q. Qin, ${ }^{12}$ X. P. Qin, ${ }^{9}$ X. S. Qin, ${ }^{41}$ Z. H. Qin, ${ }^{1,49}$ J. F. Qiu, ${ }^{1}$ S. Q. Qu, ${ }^{36}$ K. H. Rashid ${ }^{65}$ K. Ravindran, ${ }^{21}$ C. F. Redmer, ${ }^{28}$ A. Rivetti, ${ }^{66}$ V. Rodin, ${ }^{55}$

M. Rolo, ${ }^{6 c}$ G. Rong, ${ }^{1,54}$ Ch. Rosner ${ }^{15}$ M. Rump, ${ }^{60}$ H. S. Sang, ${ }^{63}$ A. Sarantsev, ${ }^{29, c}$ Y. Schelhaas, ${ }^{28}$ C. Schnier, ${ }^{4}$

K. Schoenning, ${ }^{67}$ M. Scodeggio, ${ }^{24 a, 24 b}$ D. C. Shan, ${ }^{46}$ W. Shan, ${ }^{19}$ X. Y. Shan, ${ }^{63,49}$ J. F. Shangguan, ${ }^{46}$ M. Shao, ${ }^{63,49}$ C. P. Shen, ${ }^{9}$ H. F. Shen, ${ }^{1,54}$ P. X. Shen, ${ }^{36}$ X. Y. Shen, ${ }^{1,54}$ H. C. Shi, ${ }^{63,49}$ R. S. Shi, ${ }^{1,54}$ X. Shi, ${ }^{1,49}$ X. D. Shi, ${ }^{63,49}$ J. J. Song ${ }^{41}$ W. M. Song, ${ }^{27,1}$ Y. X. Song, ${ }^{38, h}$ S. Sosio, ${ }^{66,66 c}$ S. Spataro ${ }^{66 a, 66 c}$ K. X. Su, ${ }^{68}$ P. P. Su, ${ }^{46}$ F. F. Sui, ${ }^{41}$ G. X. Sun, ${ }^{1}$ H. K. Sun, ${ }^{1}$ J. F. Sun, ${ }^{16}$ L. Sun, ${ }^{68}$ S. S. Sun, ${ }^{1,54}$ T. Sun, ${ }^{1,54}$ W. Y. Sun, ${ }^{27}$ W. Y. Sun,${ }^{34}$ X. Sun,${ }^{20, i}$ Y. J. Sun ${ }^{63,49}$ Y. K. Sun, ${ }^{63,49}$ Y. Z. Sun,${ }^{1}$ Z. T. Sun, ${ }^{1}$ Y. H. Tan ${ }^{68}$ Y. X. Tan,${ }^{63,49}$ C. J. Tang, ${ }^{45}$ G. Y. Tang, ${ }^{1}$ J. Tang, ${ }^{50}$ J. X. Teng, ${ }^{63,49}$ V. Thoren, ${ }^{67}$ W. H. Tian, ${ }^{43}$ Y. T. Tian, ${ }^{25}$ I. Uman, ${ }^{53 \mathrm{~b}}$ B. Wang, ${ }^{1}$ C. W. Wang, ${ }^{35}$ D. Y. Wang, ${ }^{38, \mathrm{~h}}$ H. J. Wang, ${ }^{31, k, 1}$ H. P. Wang, ${ }^{1,54}$ K. Wang, ${ }^{1,49}$ L. L. Wang, ${ }^{1}$ M. Wang, ${ }^{41}$ M. Z. Wang, ${ }^{38, h}$ Meng Wang, ${ }^{1,54}$ W. Wang, ${ }^{50}$ W. H. Wang, ${ }^{68}$ W. P. Wang, ${ }^{63,49}$ X. Wang, ${ }^{38, h}$ X. F. Wang, ${ }^{31, k, 1}$ X. L. Wang,,${ }^{9, \mathrm{f}}$ Y. Wang, ${ }^{50}$ Y. Wang, ${ }^{63,49}$ Y. D. Wang, ${ }^{37}$ Y. F. Wang, ${ }^{1,49,54}$ Y. Q. Wang, ${ }^{1}$ Y. Y. Wang, ${ }^{31, k, 1}$ Z. Wang, ${ }^{1,49}$ Z. Y. Wang, ${ }^{1}$ Ziyi Wang, ${ }^{54}$ Zongyuan Wang, ${ }^{1,54}$ D. H. Wei, ${ }^{12}$ F. Weidner, ${ }^{60}$ S. P. Wen, ${ }^{1}$ D. J. White, ${ }^{58}$ U. Wiedner, ${ }^{4}$ G. Wilkinson, ${ }^{61}$ M. Wolke, ${ }^{67}$ L. Wollenberg, ${ }^{4}$ J. F. Wu ${ }^{1,54}$ L. H. Wu, ${ }^{1}$ L. J. Wu, ${ }^{1,54}$ X. Wu, ${ }^{9, f} \mathrm{Z}$. Wu, ${ }^{1,49}$ L. Xia ${ }^{63,49}$ H. Xiao, ${ }^{9, f}$ S. Y. Xiao, ${ }^{1}$ Z. J. Xiao, ${ }^{34}$ X. H. Xie, ${ }^{38, h}$ Y. G. Xie, ${ }^{1,49}$ Y. H. Xie, ${ }^{6}$ T. Y. Xing, ${ }^{1,54}$ G. F. Xu, ${ }^{1}$ Q. J. Xu ${ }^{14}$ W. Xu ${ }^{1,54}$ X. P. Xu, ${ }^{46}$ Y. C. Xu, ${ }^{54}$ F. Yan,,${ }^{9, f}$ L. Yan, ${ }^{9, f}$ W. B. Yan ${ }^{63,49}$ W. C. Yan ${ }^{71}$ Xu Yan, ${ }^{46}$ H. J. Yang, ${ }^{42, e}$ H. X. Yang, ${ }^{1}$

L. Yang, ${ }^{43}$ S. L. Yang, ${ }^{54}$ Y.X. Yang, ${ }^{12}$ Yifan Yang, ${ }^{1,54}$ Zhi Yang ${ }^{25}$ M. Ye, ${ }^{1,49}$ M. H. Ye, ${ }^{7}$ J. H. Yin, ${ }^{1}$ Z. Y. You, ${ }^{50}$ B. X. Yu, ${ }^{1,49,54}$ C. X. Yu, ${ }^{36}$ G. Yu, ${ }^{1,54}$ J. S. Yu, ${ }^{20, i}$ T. Yu, ${ }^{64}$ C. Z. Yuan, ${ }^{1,54}$ L. Yuan, ${ }^{2}$ X. Q. Yuan, ${ }^{38, h}$ Y. Yuan, ${ }^{1}$ Z. Y. Yuan, ${ }^{50}$ 
C. X. Yue, ${ }^{32}$ A. A. Zafar, ${ }^{65}$ X. Zeng Zeng, ${ }^{6}$ Y. Zeng, ${ }^{20, i}$ A. Q. Zhang, ${ }^{1}$ B. X. Zhang, ${ }^{1}$ Guangyi Zhang, ${ }^{16}$ H. Zhang, ${ }^{63}$ H. H. Zhang, ${ }^{50}$ H. H. Zhang, ${ }^{27}$ H. Y. Zhang, ${ }^{1,49}$ J. J. Zhang, ${ }^{43}$ J. L. Zhang, ${ }^{69}$ J. Q. Zhang, ${ }^{34}$ J. W. Zhang, ${ }^{1,49,54}$ J. Y. Zhang, ${ }^{1}$ J. Z. Zhang, ${ }_{1,54}$ Jianyu Zhang, ${ }^{1,54}$ Jiawei Zhang, ${ }^{1,54}$ L. M. Zhang, ${ }^{52}$ L. Q. Zhang, ${ }^{50}$ Lei Zhang, ${ }^{35}$ S. Zhang, ${ }^{50}$ S. F. Zhang, ${ }^{35}$ Shulei Zhang, ${ }^{20, i}$ X. D. Zhang, ${ }^{37}$ X. Y. Zhang, ${ }^{41}$ Y. Zhang, ${ }^{61}$ Y. T. Zhang, ${ }^{71}$ Y. H. Zhang, ${ }^{1,49}$ Yan Zhang, ${ }^{63,49}$ Yao Zhang, ${ }^{1}$ Z. H. Zhang, ${ }^{6}$ Z. Y. Zhang, ${ }^{68}$ G. Zhao, ${ }^{1}$ J. Zhao, ${ }^{32}$ J. Y. Zhao, ${ }^{1,54}$ J. Z. Zhao, ${ }^{1,49}$ Lei Zhao, ${ }^{63,49}$ Ling Zhao, ${ }^{1}$ M. G. Zhao, ${ }^{36}$ Q. Zhao ${ }^{1}$ S. J. Zhao, ${ }^{71}$ Y. B. Zhao, ${ }^{1,49}$ Y.X. Zhao ${ }^{25}$ Z. G. Zhao, ${ }^{63,49}$ A. Zhemchugov, ${ }^{29, a}$ B. Zheng, ${ }^{64}$ J. P. Zheng, ${ }^{1,49}$ Y. Zheng, ${ }^{38, h}$ Y. H. Zheng, ${ }^{54}$ B. Zhong, ${ }^{34}$ C. Zhong, ${ }^{64}$ L. P. Zhou, ${ }^{1,54}$ Q. Zhou, ${ }^{1,54}$ X. Zhou, ${ }^{68}$ X. K. Zhou, ${ }^{54}$ X. R. Zhou, ${ }^{63,49}$ X. Y. Zhou, ${ }^{32}$ A. N. Zhu, ${ }^{1,54}$ J. Zhu, ${ }^{36}$ K. Zhu, ${ }^{1}$ K. J. Zhu, ${ }^{1,49,54}$ S. H. Zhu, ${ }^{62}$ T. J. Zhu, ${ }^{69}$ W. J. Zhu, ${ }^{36}$ W. J. Zhu, ${ }^{9}$, Y. C. Zhu, ${ }^{63,49}$ Z. A. Zhu, ${ }^{1,54}$ B. S. Zou, ${ }^{1}$ and J. H. Zou ${ }^{1}$

(BESIII Collaboration)

\author{
${ }^{1}$ Institute of High Energy Physics, Beijing 100049, People's Republic of China \\ ${ }^{2}$ Beihang University, Beijing 100191, People's Republic of China \\ ${ }^{3}$ Beijing Institute of Petrochemical Technology, Beijing 102617, People's Republic of China \\ ${ }^{4}$ Bochum Ruhr-University, D-44780 Bochum, Germany \\ ${ }^{5}$ Carnegie Mellon University, Pittsburgh, Pennsylvania 15213, USA \\ ${ }^{6}$ Central China Normal University, Wuhan 430079, People's Republic of China \\ ${ }^{7}$ China Center of Advanced Science and Technology, Beijing 100190, People's Republic of China \\ ${ }^{8}$ COMSATS University Islamabad, Lahore Campus, Defence Road, \\ Off Raiwind Road, 54000 Lahore, Pakistan \\ ${ }^{9}$ Fudan University, Shanghai 200443, People's Republic of China \\ ${ }^{10}$ G.I. Budker Institute of Nuclear Physics SB RAS (BINP), Novosibirsk 630090, Russia \\ ${ }^{11}$ GSI Helmholtzcentre for Heavy Ion Research GmbH, D-64291 Darmstadt, Germany \\ ${ }^{12}$ Guangxi Normal University, Guilin 541004, People's Republic of China \\ ${ }^{13}$ Guangxi University, Nanning 530004, People's Republic of China \\ ${ }^{14}$ Hangzhou Normal University, Hangzhou 310036, People's Republic of China \\ ${ }^{15}$ Helmholtz Institute Mainz, Staudinger Weg 18, D-55099 Mainz, Germany \\ ${ }^{16}$ Henan Normal University, Xinxiang 453007, People's Republic of China \\ ${ }^{17}$ Henan University of Science and Technology, Luoyang 471003, People's Republic of China \\ ${ }^{18}$ Huangshan College, Huangshan 245000, People's Republic of China \\ ${ }^{19}$ Hunan Normal University, Changsha 410081, People's Republic of China \\ ${ }^{20}$ Hunan University, Changsha 410082, People's Republic of China \\ ${ }^{21}$ Indian Institute of Technology Madras, Chennai 600036, India \\ ${ }^{22}$ Indiana University, Bloomington, Indiana 47405, USA \\ ${ }^{23 a}$ INFN Laboratori Nazionali di Frascati, INFN Laboratori Nazionali di Frascati, I-00044, Frascati, Italy \\ ${ }^{23 \mathrm{~b}}$ INFN Laboratori Nazionali di Frascati, INFN Sezione di Perugia, I-06100, Perugia, Italy \\ ${ }^{23 c}$ INFN Laboratori Nazionali di Frascati, University of Perugia, I-06100, Perugia, Italy \\ ${ }^{24 a}$ INFN Sezione di Ferrara, INFN Sezione di Ferrara, I-44122, Ferrara, Italy \\ ${ }^{24 \mathrm{~b}}$ INFN Sezione di Ferrara, University of Ferrara, I-44122, Ferrara, Italy \\ ${ }^{25}$ Institute of Modern Physics, Lanzhou 730000, People's Republic of China \\ ${ }^{26}$ Institute of Physics and Technology, Peace Ave. 54B, Ulaanbaatar 13330, Mongolia \\ ${ }^{27}$ Jilin University, Changchun 130012, People's Republic of China \\ ${ }^{28}$ Johannes Gutenberg University of Mainz, Johann-Joachim-Becher-Weg 45, D-55099 Mainz, Germany \\ ${ }^{29}$ Joint Institute for Nuclear Research, 141980 Dubna, Moscow region, Russia \\ ${ }^{30}$ Justus-Liebig-Universitaet Giessen, II. Physikalisches Institut, Heinrich-Buff-Ring 16, \\ D-35392 Giessen, Germany \\ ${ }^{31}$ Lanzhou University, Lanzhou 730000, People's Republic of China \\ ${ }^{32}$ Liaoning Normal University, Dalian 116029, People's Republic of China \\ ${ }^{33}$ Liaoning University, Shenyang 110036, People's Republic of China \\ ${ }^{34}$ Nanjing Normal University, Nanjing 210023, People's Republic of China \\ ${ }^{35}$ Nanjing University, Nanjing 210093, People's Republic of China \\ ${ }^{36}$ Nankai University, Tianjin 300071, People's Republic of China \\ ${ }^{37}$ North China Electric Power University, Beijing 102206, People's Republic of China \\ ${ }^{38}$ Peking University, Beijing 100871, People's Republic of China \\ ${ }^{39}$ Qufu Normal University, Qufu 273165, People's Republic of China \\ ${ }^{40}$ Shandong Normal University, Jinan 250014, People's Republic of China \\ ${ }^{41}$ Shandong University, Jinan 250100, People's Republic of China \\ ${ }^{42}$ Shanghai Jiao Tong University, Shanghai 200240, People's Republic of China
}




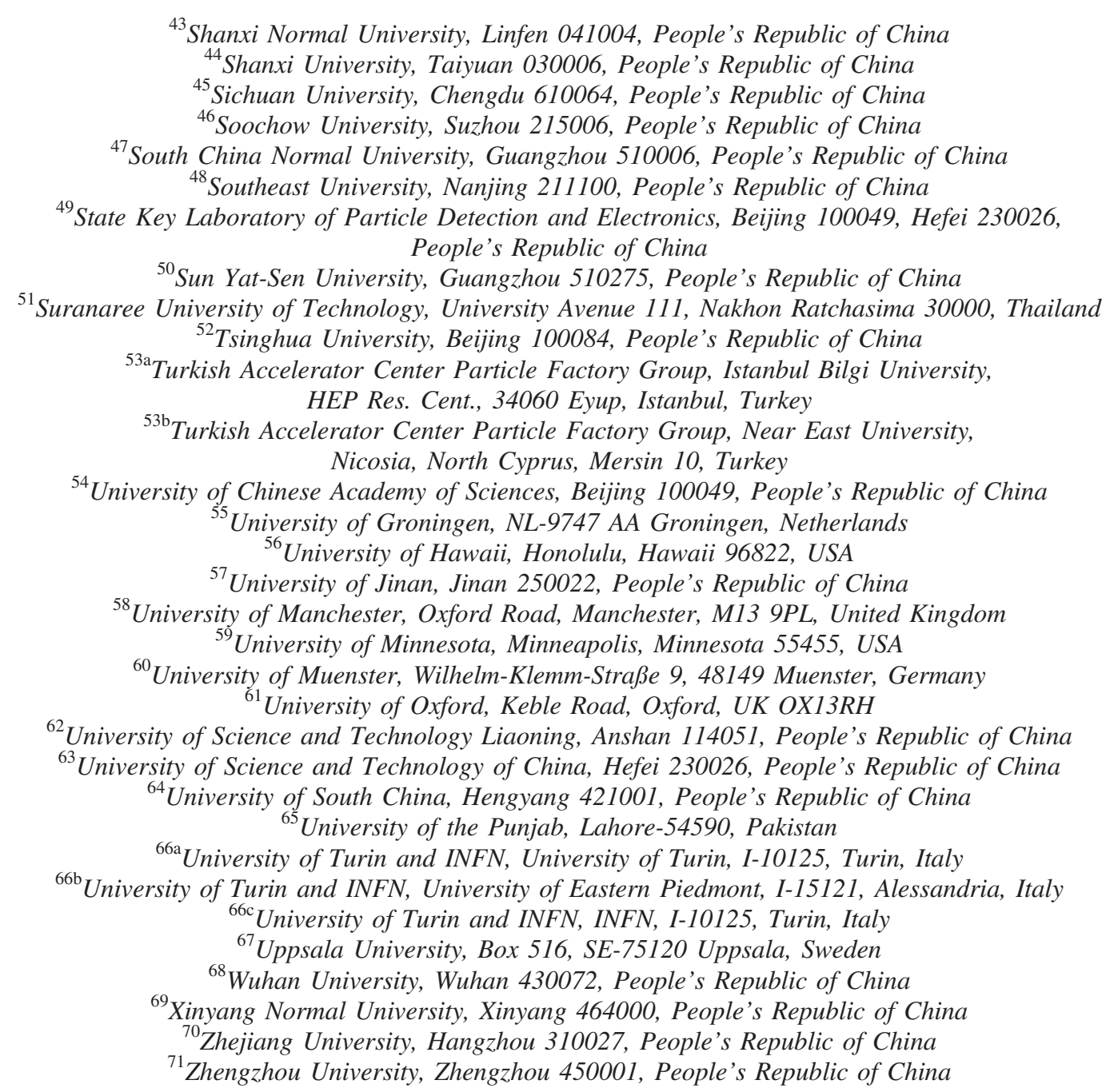

(Received 16 April 2021; accepted 8 June 2021; published 9 July 2021)

We measure the inclusive semielectronic decay branching fraction of the $D_{s}^{+}$meson. A double-tag technique is applied to $e^{+} e^{-}$annihilation data collected by the BESIII experiment at the BEPCII collider, operating in the center-of-mass energy range 4.178-4.230 GeV. We select positrons from $D_{s}^{+} \rightarrow X e^{+} \nu_{e}$

\footnotetext{
${ }^{a}$ Also at the Moscow Institute of Physics and Technology, Moscow 141700, Russia.

${ }^{\mathrm{b}}$ Also at the Novosibirsk State University, Novosibirsk 630090, Russia.

"Also at the NRC "Kurchatov Institute," PNPI, 188300 Gatchina, Russia.

${ }^{\mathrm{d}}$ Also at Goethe University Frankfurt, 60323 Frankfurt am Main, Germany.

eAlso at Key Laboratory for Particle Physics, Astrophysics and Cosmology, Ministry of Education; Shanghai Key Laboratory for Particle Physics and Cosmology; Institute of Nuclear and Particle Physics, Shanghai 200240, People's Republic of China.

${ }^{\mathrm{f}}$ Also at Key Laboratory of Nuclear Physics and Ion-beam Application (MOE) and Institute of Modern Physics, Fudan University, Shanghai 200443, People's Republic of China.

${ }^{\mathrm{g}}$ Also at Harvard University, Department of Physics, Cambridge, Massachusetts 02138, USA.

${ }^{\mathrm{h}}$ Also at State Key Laboratory of Nuclear Physics and Technology, Peking University, Beijing 100871, People's Republic of China.

${ }^{\mathrm{i}}$ Also at School of Physics and Electronics, Hunan University, Changsha 410082, People's Republic of China.

${ }^{j}$ Also at Guangdong Provincial Key Laboratory of Nuclear Science, Institute of Quantum Matter, South China Normal University, Guangzhou 510006, People's Republic of China.

${ }^{k}$ Also at Frontiers Science Center for Rare Isotopes, Lanzhou University, Lanzhou 730000, People's Republic of China.

${ }^{1}$ Also at Lanzhou Center for Theoretical Physics, Lanzhou University, Lanzhou 730000, People's Republic of China.

${ }^{\mathrm{m}}$ Currently at Istinye University, 34010 Istanbul, Turkey.
}

Published by the American Physical Society under the terms of the Creative Commons Attribution 4.0 International license. Further distribution of this work must maintain attribution to the author(s) and the published article's title, journal citation, and DOI. Funded by $S C O A P^{3}$. 
with momenta greater than $200 \mathrm{MeV} / c$ and determine the laboratory momentum spectrum, accounting for the effects of detector efficiency and resolution. The total positron yield and semielectronic branching fraction are determined by extrapolating this spectrum below the momentum cutoff. We measure the $D_{s}^{+}$ semielectronic branching fraction to be $(6.30 \pm 0.13$ (stat.) \pm 0.09 (syst.) \pm 0.04 (ext.) $) \%$, showing no evidence for unobserved exclusive semielectronic modes. We combine this result with external data taken from literature to determine the ratio of the $D_{s}^{+}$and $D^{0}$ semielectronic widths, $\frac{\Gamma\left(D_{s}^{+} \rightarrow X e^{+} \nu_{e}\right)}{\Gamma\left(D^{0} \rightarrow X e^{+} \nu_{e}\right)}=$ $0.790 \pm 0.016$ (stat.) \pm 0.011 (syst.) \pm 0.016 (ext.). Our results are consistent with and more precise than previous measurements.

DOI: 10.1103/PhysRevD.104.012003

\section{INTRODUCTION}

The $D_{s}^{+}$meson is the ground state of charmed-strange mesons, and precise measurements of its semileptonic decays allow for crucial tests of Standard Model predictions of flavor-changing interactions. This article presents a new measurement of the $D_{s}^{+}$-meson inclusive semielectronic branching fraction and positron momentum spectrum. (Here and throughout this article, charge conjugate modes are implied.) A previous measurement by the CLEO-c experiment reported the first results for these quantities, including the measurement of $\mathcal{B}\left(D_{s}^{+} \rightarrow X e^{+} \nu_{e}\right)=$ $(6.52 \pm 0.39$ (stat.) \pm 0.15 (syst.) $) \% \quad[1]$. Measuring this branching fraction with improved precision contributes to a comprehensive understanding of $D_{s}^{+}$decays and is an important component of the overall experimental and theoretical heavy-flavor physics program.

Table I lists the six exclusive $D_{s}^{+}$semielectronic modes that have been observed to date and their branching fractions, as well as the branching fraction of $D_{s}^{+} \rightarrow$ $\tau^{+} \nu_{\tau} \rightarrow e^{+} \nu_{e} \nu_{\tau} \bar{\nu}_{\tau}$ and the previously measured $D_{s}^{+} \rightarrow$ $X e^{+} \nu_{e}$ branching fraction [1-15]. By comparing the inclusive semielectronic branching fraction with the sum of all measured exclusive semielectronic branching fractions, we can estimate the branching fraction for further unobserved $D_{s}^{+}$semielectronic decays. Measurements of the semielectronic branching fractions for different charmed mesons can be combined to probe for nonspectator effects in heavy-meson decays [16,17]. The CLEO-c measurement of the ratio of the $D_{s}^{+}$and $D^{0}$ semielectronic widths, $\frac{\Gamma\left(D_{s}^{+} \rightarrow X e^{+} \nu_{e}\right)}{\Gamma\left(D^{0} \rightarrow X e^{+} \nu_{e}\right)}=0.815 \pm 0.052$, is in agreement with predictions employing an effective quark model [18] and shows that nonspectator effects are present in semielectronic charmed-meson decays. It has also been demonstrated with CLEO-c data that the inclusive semielectronic momentum spectrum can be used to make sensitive tests for specific nonspectator processes, such as weak annihilation $[19,20]$. Precise understanding of these processes is required for application of heavy-quark expansion in extracting Cabibbo-Kobayashi-Maskawa (CKM) matrix elements from inclusive semileptonic $B$ meson decays [21]. Thus, the improved precision of both the inclusive branching fraction and the momentum spectrum of $D_{s}^{+}$ semielectronic decays reported in this article have the potential to contribute to reducing theoretical uncertainties in determining CKM parameters with heavy-meson decays.

The remainder of the article is organized in seven sections. The BESIII detector, the analyzed data, and the Monte Carlo (MC) simulation samples are described in Sec. II. An overview of the measurement technique is presented in Sec. III. Event selection requirements based on full reconstruction of hadronic $D_{s}^{-}$decays are discussed in Sec. IV. Semielectronic decay selection requirements and further analysis of candidate signal events are presented in Sec. V. The systematic uncertainties of our measurement are evaluated in Sec. VI. We conclude with a summary of our results in Sec. VII.

\section{DETECTOR AND DATA SAMPLES}

The BESIII detector records the results of symmetric $e^{+} e^{-}$collisions provided by the BEPCII collider [22]. BEPCII produces collisions at center-of-mass energies $\left(E_{\mathrm{cm}}\right)$ between 2 and $4.9 \mathrm{GeV}$, and BESIII has collected the world's largest data samples near a number of pairproduction threshold energies for charmed hadrons. The BESIII detector is composed of the following subsystems for particle detection and identification: a helium-based multilayer drift chamber (MDC), a plastic scintillator timeof-flight system (TOF), a CsI(Tl) electromagnetic calorimeter (EMC), a 1.0 T superconducting solenoid, and a set of resistive plate chambers for muon identification. The acceptance of the BESIII detector for charged particles is $93 \%$ of the full solid angle. Ionization energy deposits in the MDC are used to reconstruct charged particle tracks and determine particle momenta from the curvature in the magnetic field. The MDC provides a momentum resolution of $0.5 \%$ for particles with a momentum of $1 \mathrm{GeV} / c$. The end cap TOF system was upgraded in 2015 with multigap resistive plate chamber technology, providing a time resolution of 60 picoseconds [23]. Measurements of charged particle specific ionization in the MDC $(d E / d x)$, the flight time in the TOF, and the energy deposit in the EMC associated with a track are combined to identify particles. A detailed description of the BESIII detector is given in Ref. [24].

Inclusive MC samples with the equivalent of five times the luminosity of data and exclusive MC samples with the 
TABLE I. Branching fractions for observed $D_{s}^{+}$semielectronic decays and for $D_{s}^{+} \rightarrow \tau^{+} \nu_{\tau} \rightarrow e^{+} \nu_{e} \nu_{\tau} \bar{\nu}_{\tau}$. The listed uncertainties are the total uncertainties. The $D_{s}^{+} \rightarrow f_{0}(980) e^{+} \nu_{e}, f_{0}(980) \rightarrow$ $\pi \pi$ branching fraction is calculated based on the measurements of $D_{s}^{+} \rightarrow \pi^{+} \pi^{-} e^{+} \nu_{e}$ from [2] and corrected for the corresponding $\pi^{0} \pi^{0}$ branching fraction by an isospin correction factor of $\frac{3}{2}$.

\begin{tabular}{lcc}
\hline \hline Mode & Averaged branching fraction \\
\hline$D_{s}^{+} \rightarrow \phi e^{+} \nu_{e}$ & $(2.37 \pm 0.11) \%$ & {$[3-6]$} \\
$D_{s}^{+} \rightarrow \eta e^{+} \nu_{e}$ & $(2.32 \pm 0.08) \%$ & {$[6-8]$} \\
$D_{s}^{+} \rightarrow \eta^{\prime} e^{+} \nu_{e}$ & $(0.80 \pm 0.07) \%$ & {$[6-8]$} \\
$D_{s}^{+} \rightarrow K^{0} e^{+} \nu_{e}$ & $(0.34 \pm 0.04) \%$ & {$[6,9]$} \\
$D_{s}^{+} \rightarrow K^{*}(892)^{0} e^{+} \nu_{e}$ & $(0.21 \pm 0.03) \%$ & {$[6,9]$} \\
$D_{s}^{+} \rightarrow f_{0}(980) e^{+} \nu_{e}, f_{0}(980) \rightarrow \pi \pi$ & $(0.30 \pm 0.05) \%$ & {$[2]$} \\
Sum of semielectronic modes & $(6.34 \pm 0.17) \%$ & \\
$\mathcal{B}\left(D_{s}^{+} \rightarrow X e^{+} \nu_{e}\right)$ & $(6.52 \pm 0.42) \%$ & {$[1]$} \\
$D_{s}^{+} \rightarrow \tau^{+} \nu_{\tau} \rightarrow e^{+} \bar{\nu}_{\tau} \nu_{e} \nu_{\tau}$ & $(0.96 \pm 0.04) \%$ & {$[10-15]$} \\
\hline \hline
\end{tabular}

equivalent of 35 times the luminosity of data are used to optimize selection criteria, investigate distributions of signal and background processes, and determine the efficiencies of our selection criteria. Hadronic simulation samples are produced using the event generators KKMC [25] and EvtGen [26,27]. BabaYagaNLO [28] is used to produce radiative Bhabha $\left(e^{+} e^{-} \rightarrow \gamma e^{+} e^{-}\right)$samples. Final-state radiation (FSR) of particles is simulated with PнОтоS [29]. Interaction of the simulated particles with the detector material is handled by GEANT4 [30], which uses a detailed XML-based description of the detector geometry [31].

In 2016, BESIII collected $3.19 \mathrm{fb}^{-1}$ of data at $E_{\mathrm{cm}}=$ $4.178 \mathrm{GeV}$, which provides approximately $6.4 \times 10^{6} D_{s}^{+}$ mesons primarily through the process $e^{+} e^{-} \rightarrow D_{s}^{*+} D_{s}^{-}$, with a small contribution from $e^{+} e^{-} \rightarrow D_{s}^{+} D_{s}^{-}$. We analyze the entirety of this sample, as well as $2.08 \mathrm{fb}^{-1}$ of data collected in 2017 in the $E_{\mathrm{cm}}$ range 4.189-4.219 GeV in 2017 and $1.05 \mathrm{fb}^{-1}$ of data collected in the $E_{\mathrm{cm}}$ range 4.225-4.230 GeV in 2013. These three samples are analyzed separately due to differing detector and running conditions. A summary of the datasets with their $E_{\mathrm{cm}}$ [32], integrated luminosities [33], and estimated number of $D_{s}^{+}$ mesons produced is shown in Table II.

TABLE II. $E_{\mathrm{cm}}$, integrated luminosities, and estimated number of $D_{s}$ mesons $\left(N_{D_{s}}\right)$ for the analyzed data samples. In each case, the first listed uncertainty is statistical, and the second is systematic.

\begin{tabular}{lcc}
\hline \hline$E_{\mathrm{cm}}(\mathrm{MeV})$ & $\int \mathcal{L} d t\left(\mathrm{pb}^{-1}\right)$ & $N_{D_{s}}\left(\times 10^{6}\right)$ \\
\hline 4178 & $3189.0 \pm 0.9 \pm 31.9$ & 6.4 \\
4189 & $526.7 \pm 0.1 \pm 2.2$ & 1.0 \\
4199 & $526.0 \pm 0.1 \pm 2.1$ & 1.0 \\
4209 & $517.1 \pm 0.1 \pm 1.8$ & 0.9 \\
4219 & $514.6 \pm 0.1 \pm 1.8$ & 0.8 \\
$4225-4230[32]$ & $1047.3 \pm 0.1 \pm 10.2[33]$ & 1.3 \\
\hline \hline
\end{tabular}

\section{MEASUREMENT TECHNIQUE}

Our analysis procedure employs the double-tag technique pioneered by the MARKIII Collaboration [34]. We fully reconstruct hadronic $D_{s}^{-}$mesons (the tag or tag side) and determine the number of signal events by analyzing the remaining charged tracks unused in the reconstruction of the tags (the recoil or recoil side). We refer to events where a tag meson is found as single-tag events and events where a semielectronic decay is identified on the recoil side in addition to the tag meson as double-tag events. Our singletag selection is described in more detail in Sec. IV.

Since identification and reconstruction of positron tracks is only possible above a certain momentum threshold with the BESIII detector, double-tag candidate tracks are only accepted with $p>200 \mathrm{MeV} / c$. The differential inclusive semielectronic branching fraction as a function of the electron momentum $p_{e}$ is given as

$\frac{d \mathcal{B}_{\mathrm{SL}}}{d p_{e}}=\frac{\Delta\left(\frac{n_{\mathrm{DT}}}{\epsilon_{\mathrm{DT}}}\right) / \Delta p_{e}}{n_{\mathrm{ST}} / \epsilon_{\mathrm{ST}}}=\frac{\left(\frac{\Delta n_{\mathrm{DT}}}{\epsilon_{\mathrm{Sig}}}\right) / \Delta p_{e}}{n_{\mathrm{ST}} \frac{\epsilon_{\mathrm{ST}}^{\prime}}{\epsilon_{\mathrm{ST}}}}=\frac{\Delta N_{\mathrm{DT}} / \Delta p_{e}}{n_{\mathrm{ST}} b_{\mathrm{tag}}}$.

In this equation, $n_{\mathrm{ST}}$ is the number of the observed single-tag events, $\Delta n_{\mathrm{DT}}$ is the number of the observed (semielectronic) double-tag events in a particular $p_{e}$ bin, and $\epsilon_{\mathrm{ST}}$ and $\epsilon_{\mathrm{DT}}$ are the single-tag and double-tag reconstruction efficiencies, respectively. We define $\epsilon_{\mathrm{DT}}=\epsilon_{\mathrm{ST}}^{\prime} \times \epsilon_{\text {sig }}$, where $\epsilon_{\text {sig }}$ is the momentum-dependent efficiency of reconstructing only the signal side and $\epsilon_{\mathrm{ST}}^{\prime}$ is the tag-side reconstruction efficiency given that a signal event is present on the recoil side. We define $b_{\text {tag }} \equiv \frac{\epsilon_{S T}^{\prime}}{\epsilon_{\mathrm{ST}}}$ as the tag bias, which accounts for the difference in the single-tag detection efficiency given that a semielectronic decay is present on the recoil side and $\Delta N_{\mathrm{DT}}$ as the true number of double-tag signal events in our singletag sample for a particular $p_{e}$ bin.

We begin our signal selection by sorting recoil-side tracks with $p>200 \mathrm{MeV} / c$ into momentum bins. For a given momentum bin, we further sort these tracks by their charge: tracks with charge opposite to the tag [right sign (RS)] and tracks with the same charge as the tag [wrong sign (WS)]. The WS sample is used to estimate chargesymmetric backgrounds in the RS distributions, which include the true signal. For both charge categories in each momentum bin, we sort tracks into three mutually exclusive particle identification hypotheses: electron/positron ( $e$ ID), pion $(\pi \mathrm{ID})$, and kaon $(K \mathrm{ID})$. The similar detector response to muons and pions allows us to treat muons as identical to pions with negligible uncertainty. For each of these six categories in a given momentum bin, we determine the number of tracks that originate from a true $D_{s}^{+}$meson by fitting to the invariant-mass distribution of the tag $D_{s}^{-}$ candidate.

We relate the true number of double-tag signal events in our single-tag sample and the observed number of 
signal-candidate tracks from double-tag events through detector response matrices for charged particle tracking $\left(A_{\text {trk }}\right)$ and particle identification (PID) $\left(A_{\mathrm{PID}}\right)$ :

$n_{\mathrm{DT}}^{b}\left(p_{i}\right)=\sum_{a} A_{\mathrm{PID}}\left(b \mid a, p_{i}\right) \sum_{j} A_{\mathrm{trk}}\left(a \mid p_{i}, p_{j}\right) N_{\mathrm{DT}}^{a}\left(p_{j}\right)$.

Here, $A_{\text {trk }}\left(a \mid p_{i}, p_{j}\right)$ gives the probability of a particle of type $a(a \in\{e, \pi, K\})$ tracked in momentum bin $p_{i}$ to have true momentum in bin $p_{j}$. Similarly, $A_{\mathrm{PID}}\left(b \mid a, p_{i}\right)$ gives the probability of a particle of type $a$ passing the PID requirements of particle type $b(b \in\{e, \pi, K\})$ in track momentum bin $p_{i}$.

We determine the observed double-tag yields $n_{\mathrm{DT}}^{b}\left(p_{i}\right)$ by fitting the invariant-mass distribution of tag-side mesons. For both RS and WS tracks in each momentum bin, we account for $e$ ID efficiencies and particles misidentified as positrons with the PID unfolding method introduced in Eq. (2):

$$
\begin{aligned}
{\left[\begin{array}{c}
n_{\text {trk }}^{e} \\
n_{\text {trk }}^{\pi} \\
n_{\text {trk }}^{K}
\end{array}\right] } & =A_{\mathrm{PID}}^{-1}\left[\begin{array}{c}
n_{\mathrm{DT}}^{e} \\
n_{\mathrm{DT}}^{\pi} \\
n_{\mathrm{DT}}^{K}
\end{array}\right], \\
A_{\mathrm{PID}} & =\left[\begin{array}{ccc}
\epsilon_{e} & \mathrm{P}_{\pi \rightarrow e} & P_{K \rightarrow e} \\
P_{e \rightarrow \pi} & \epsilon_{\pi} & P_{K \rightarrow \pi} \\
P_{e \rightarrow K} & P_{\pi \rightarrow K} & \epsilon_{K}
\end{array}\right] .
\end{aligned}
$$

Here, $\epsilon_{a}$ is the efficiency of our PID selection requirements for the particles of species $a$, and $P_{a \rightarrow b}$ is the probability of a particle of species $a$ passing the selection requirements for a particle of species $b$. We apply this PID unfolding to the fitted RS and WS yields for each momentum bin and take the $e$ solution to determine the number of tracked positrons originating from a $D_{s}^{+}$meson. We then take the difference of the number of tracked RS positrons and WS electrons to subtract the contributions of positrons from Dalitz decays of light mesons in the final state of $D_{s}^{+}$decay such as $\pi^{0} \rightarrow \gamma e^{+} e^{-}$and any other charge-symmetric backgrounds.

This leaves us with the momentum spectrum of tracked positrons from $D_{s}^{+} \rightarrow X e^{+} \nu_{e}$ events and a smaller contribution from $D_{s}^{+} \rightarrow \tau^{+} \nu_{\tau} \rightarrow e^{+} \nu_{e} \nu_{\tau} \bar{\nu}_{\tau}$. To account for tracking inefficiency and mismeasurement, we execute the second unfolding from Eq. (2):

$$
N_{\mathrm{DT}}\left(p_{j}\right)=A_{\mathrm{trk}}^{-1} n_{\mathrm{trk}}^{e}\left(p_{i}\right) .
$$

This provides the true sum of $D_{s}^{+} \rightarrow X e^{+} \nu_{e}$ and $D_{s}^{+} \rightarrow$ $\tau^{+} \nu_{\tau} \rightarrow e^{+} \nu_{e} \nu_{\tau} \bar{\nu}_{\tau}$ positron momentum spectra. We then subtract the contribution of $D_{s}^{+} \rightarrow \tau^{+} \nu_{\tau} \rightarrow e^{+} \nu_{e} \nu_{\tau} \bar{\nu}_{\tau}$ based on the branching fraction in Table I to obtain the $D_{s}^{+} \rightarrow$ $X e^{+} \nu_{e}$ momentum spectrum for $p_{e}>200 \mathrm{MeV} / c$. This procedure was validated with five data-sized MC samples and found to recover the correct yields.
To account for positrons with $p_{e} \leq 200 \mathrm{MeV} / c$, we fit the expected $D_{s}^{+} \rightarrow X e^{+} \nu_{e}$ momentum spectrum with an assumed spectrum, described in more detail in Sec. V, and add the fitted yields in the region $p_{e} \leq 200 \mathrm{MeV} / c$ to the measured yields with $p_{e}>200 \mathrm{MeV} / c$. This gives us the signal-efficiency-corrected number of signal events

$$
N_{\mathrm{DT}}=N_{p_{e}>200 \mathrm{MeV} / c}+N_{p_{e} \leq 200 \mathrm{MeV} / c},
$$

which allows us to calculate the branching fraction similarly to Eq. (1) through

$$
\mathcal{B}\left(D_{s}^{+} \rightarrow X e^{+} \nu_{e}\right)=\frac{N_{\mathrm{DT}}}{n_{\mathrm{ST}} b_{\mathrm{tag}}},
$$

with the MC simulation prediction of $b_{\text {tag }}$ and the determined number of single-tag events $n_{\mathrm{ST}}$.

\section{SINGLE-TAG EVENT SELECTION}

We select single-tag candidates using only the $D_{s}^{-} \rightarrow$ $K^{+} K^{-} \pi^{-}$hadronic decay mode because it is unique in having sufficient statistics and well-known backgrounds. All tag candidate decay products are required to satisfy the following track-quality requirements: the track must originate from a region within $1(10) \mathrm{cm}$ of the $e^{+} e^{-}$interaction point perpendicular (parallel) to the $z$ axis, which is the symmetry axis of the MDC, and must be tracked with an angle $\theta$ with respect to the $z$ axis that satisfies $|\cos \theta|<0.93$. We apply $\pi / K$ ID based on $d E / d x$ and TOF measurements to all tag-candidate daughters to maximize the purity of the tag sample. Multiple candidates in a single event are allowed, both to increase the single-tag selection efficiency and to minimize the tag bias, $b_{\text {tag }}$. We calculate the recoil mass against the tag $D_{s}^{-}$candidate,

$M_{\mathrm{Rec}} c^{2}=\left[\left(E_{\mathrm{cm}}-\sqrt{\left|\vec{p}_{D_{s}} c\right|^{2}+m_{D_{s}}^{2} c^{4}}\right)^{2}-\left|\vec{p}_{D_{s}} c\right|^{2}\right]^{\frac{1}{2}}$,

where $\vec{p}_{D_{s}}$ is the momentum of the reconstructed tag $D_{s}^{-}$ candidate and $m_{D_{s}}$ is the known $D_{s}$ mass [35]. We require the recoil mass to be consistent with a $D_{s}^{*+} D_{s}^{-}$event hypothesis by imposing requirements for each dataset: $2057<M_{\mathrm{Rec}}<2177 \mathrm{MeV} / c^{2}$ for $E_{\mathrm{cm}}=4.178 \mathrm{GeV}, 2145<$ $M_{\mathrm{Rec}}<2190 \mathrm{MeV} / c^{2}$ for $E_{\mathrm{cm}}=4.189-4.219 \mathrm{GeV}$, and $2150<M_{\mathrm{Rec}}<2200 \mathrm{MeV} / c^{2}$ for $E_{\mathrm{cm}}=4.225-4.230 \mathrm{GeV}$. For about $20 \%$ of cases, our event selection identifies more than one candidate per event after these recoil-mass requirements. Background due to extra candidates is subtracted as part of the fitting procedure described later in this section. The $M_{\text {Rec }}$ distributions for each dataset can be seen in Fig. 1 . While differences between the MC simulation and data can be seen in the figure, our measurement is not sensitive to the MC simulation of this distribution.

We determine the number of true single-tag candidates by performing an unbinned fit to the distribution of the 



FIG. 1. Recoil-mass distribution of $D_{s}^{-} \rightarrow K^{+} K^{-} \pi^{-}$candidates from the $E_{\mathrm{cm}}=4.178 \mathrm{GeV}$ (upper), $E_{\mathrm{cm}}=4.189-4.219 \mathrm{GeV}$ (middle), and $E_{\mathrm{cm}}=4.225-4.230 \mathrm{GeV}$ (lower) datasets. The black points with error bars are data, and the black and red histograms are the signal and background distributions predicted by the MC simulation, scaled by integrated luminosity. The dashed red lines indicate the selection conditions.

invariant mass of the tag $D_{s}^{-}$candidates, $M_{\text {Inv }}$. The signal shape is based on MC simulation and convolved with a Gaussian function whose width and mean are left free in the fit to account for a possible difference in resolution and calibration between data and the MC simulation. The distribution of backgrounds in this variable is modeled using a second-order Chebyshev polynomial. The fit range is chosen to be within $\pm 40 \mathrm{MeV} / c^{2}$ of the known $D_{s}^{+}$mass for the $E_{\mathrm{cm}}=4.178 \mathrm{GeV}$ distribution corresponding to approximately $5 \sigma$ of the simulated $M_{\text {Inv }}$ distribution. In the
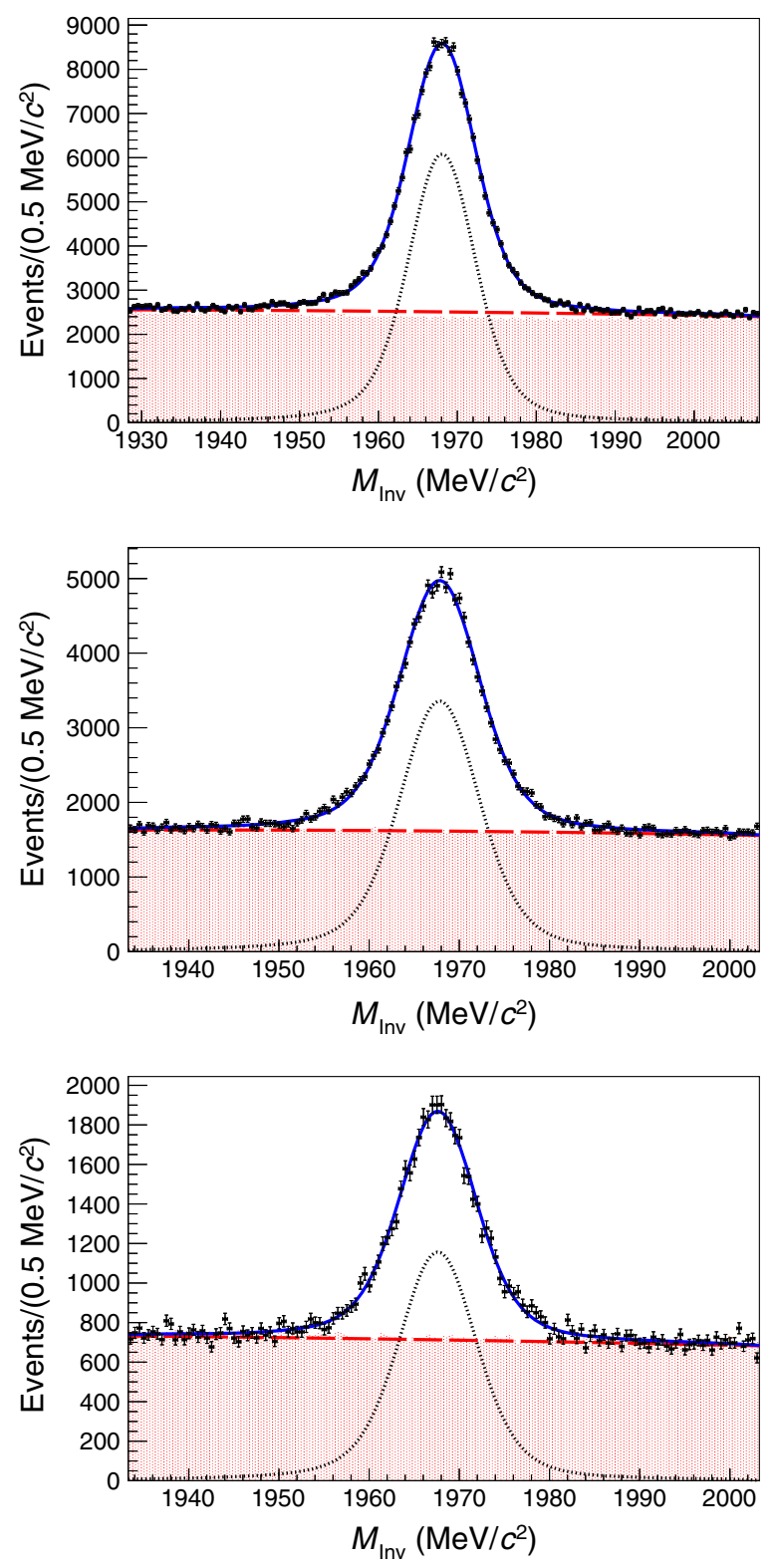

FIG. 2. Results of fits to invariant-mass distributions of $D_{s}^{-} \rightarrow$ $K^{+} K^{-} \pi^{-}$single-tag candidates from the $E_{\mathrm{cm}}=4.178 \mathrm{GeV}$ (upper), $\quad E_{\mathrm{cm}}=4.189-4.219 \mathrm{GeV}$ (middle), and $E_{\mathrm{cm}}=$ 4.225-4.230 GeV (lower) datasets. In each plot, the solid blue line is the result of the total fit, the dashed red line is the fitted distribution of non- $D_{s}^{-} \rightarrow K^{+} K^{-} \pi^{-}$backgrounds, the dotted black line is the fitted signal distribution, the filled red histogram is the contribution from backgrounds predicted by MC simulation, and the black points with error bars are data. The choice of binning is arbitrary and used solely for display. MC simulation distributions are scaled by luminosity.

$E_{\mathrm{cm}}=4.189-4.219 \mathrm{GeV}$ and $E_{\mathrm{cm}}=4.225-4.230 \mathrm{GeV}$ datasets, the fit range is reduced to be within $\pm 35 \mathrm{MeV} / c^{2}$ of the known $D_{s}$ mass due to nonpolynomial background structures appearing at the edges of the fit range. The single-tag fits to each dataset are shown in Fig. 2, and the fitted yields are listed in Table III. 
TABLE III. Fitted single-tag yields from each dataset. Only statistical uncertainties are shown.

\begin{tabular}{lc}
\hline \hline Dataset & Fitted single-tag yields \\
\hline$E_{\mathrm{cm}}=4.178 \mathrm{GeV}$ & $147581 \pm 779$ \\
$E_{\mathrm{cm}}=4.189-4.219 \mathrm{GeV}$ & $85845 \pm 705$ \\
$E_{\mathrm{cm}}=4.225-4.230 \mathrm{GeV}$ & $29234 \pm 435$ \\
Sum & $262660 \pm 1137$ \\
\hline \hline
\end{tabular}

The $E_{\mathrm{cm}}=4.178 \mathrm{GeV}$ dataset provides approximately $56 \%$ of our single-tag sample, and the $E_{\mathrm{cm}}=4.189$ $4.219 \mathrm{GeV}$ and $E_{\mathrm{cm}}=4.225-4.230 \mathrm{GeV}$ datasets provide $33 \%$ and $11 \%$, respectively. Within each dataset, $99 \%$ of our $D_{s}^{-}$candidates comes from $D_{s}^{*} D_{s}$ events, and $1 \%$ comes from $D_{s} D_{s}$ events. $D_{s}^{*} D_{s}^{*}$ events can be produced at $E_{\mathrm{cm}}=4.225-4.230 \mathrm{GeV}$, but contribute negligibly to our candidate sample.

The single-tag reconstruction efficiency depends on the topology of the recoil-side decay, which we account for through $b_{\text {tag }}$. We determine the tag bias from MC samples for double-tag events from each of the observed semielectronic modes listed in Table I and for $D_{s}^{+} \rightarrow$ $\tau^{+} \nu_{\tau} \rightarrow e^{+} \nu_{e} \nu_{\tau} \bar{\nu}_{\tau}$. The $D_{s}^{+} \rightarrow X e^{+} \nu_{e}$ tag bias is determined by averaging the tag biases for the six observed modes weighted by the branching fractions in Table I. The singletag efficiencies with no specification on the signal-side and the determined tag biases for $D_{s}^{+} \rightarrow X e^{+} \nu_{e}$ and $D_{s}^{+} \rightarrow$ $\tau^{+} \nu_{\tau} \rightarrow e^{+} \nu_{e} \nu_{\tau} \bar{\nu}_{\tau}$ for each dataset are shown in Table IV.

\section{DOUBLE-TAG SELECTION AND ANALYSIS}

After a single-tag candidate is found, we begin searching for positron candidates among the recoil-side tracks. We sort recoil-side tracks that satisfy track requirements defined in Sec. IV into $1850-\mathrm{MeV} / c$ momentum bins between $200 \mathrm{MeV} / c$ and $1100 \mathrm{MeV} / c$. Mutually exclusive PID hypotheses are assigned to each track based on information from the MDC, TOF, and EMC.

Based on our PID assignment, we fill tag-side invariantmass distributions in each momentum bin for each of the six categories of recoil-side tracks defined in Sec. III. We determine the number of tracks in each category originating from true $D_{s}^{+}$events by performing an unbinned fit to the tag-side invariant-mass distribution with the same signal shape used for the single-tag fit, with the Gaussian
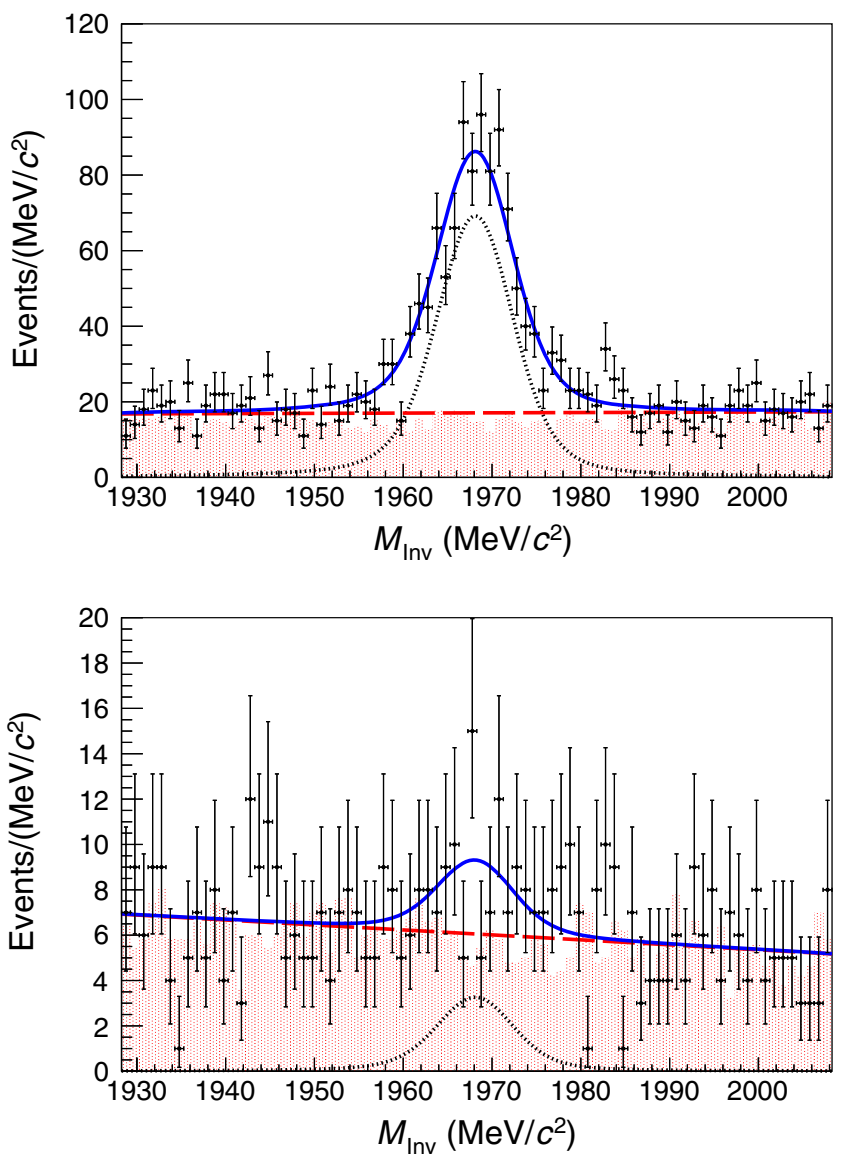

FIG. 3. Example tag invariant-mass fits for double-tag RS (upper) and WS (lower) positron candidates with momentum in the range $450-500 \mathrm{MeV} / c$ from $E_{\mathrm{cm}}=4.178 \mathrm{GeV}$ data. In both plots, the solid blue line is the result of the total fit, the dashed red line is the fitted distribution of non- $D_{s}^{-} \rightarrow K^{+} K^{-} \pi^{-}$backgrounds, the dotted black line is the fitted signal distribution, the filled red histogram is the contribution from backgrounds predicted by MC simulation, and the black points with error bars are data. The choice of binning is arbitrary and used solely for display. MC simulation distributions are scaled by the number of single-tag events.

parameters fixed to those determined in the single-tag fit. We employ a first-order Chebyshev polynomial to model the distribution of backgrounds. Two examples of the 324 fits we perform are shown in Fig. 3. The full set of fits is made available as Supplemental Material [36].

TABLE IV. Single-tag efficiencies for all events $\left(\epsilon_{\mathrm{ST}}\right)$ and tag bias for $D_{s}^{+} \rightarrow X e^{+} \nu_{e}$ events $\left(b_{\mathrm{tag}}\right)$ and for $D_{s}^{+} \rightarrow$ $\tau^{+} \nu_{\tau} \rightarrow e^{+} \nu_{e} \nu_{\tau} \bar{\nu}_{\tau}$ events $\left(b_{\text {tag }}^{\tau}\right)$ from each dataset determined with MC samples. Listed uncertainties are only statistical.

\begin{tabular}{lccr}
\hline \hline Dataset & $\epsilon_{\mathrm{ST}}$ & $b_{\mathrm{tag}}$ & $b_{\mathrm{tag}}^{\tau}$ \\
\hline$E_{\mathrm{cm}}=4.178 \mathrm{GeV}$ & $(43.10 \pm 0.01) \%$ & $1.007 \pm 0.001$ & $1.037 \pm 0.003$ \\
$E_{\mathrm{cm}}=4.189-4.219 \mathrm{GeV}$ & $(42.46 \pm 0.02) \%$ & $1.004 \pm 0.002$ & $1.034 \pm 0.004$ \\
$E_{\mathrm{cm}}=4.225-4.230 \mathrm{GeV}$ & $(40.48 \pm 0.03) \%$ & $1.005 \pm 0.003$ & $1.044 \pm 0.007$ \\
\hline \hline
\end{tabular}


Measured yields as a function of momentum bin for each category are shown in Fig. 4.

With the yields determined from the fits, we perform the matrix unfolding procedure described in Sec. III to correct for the inefficiencies of our electron/positron identification and for the misidentification of pions and kaons as electrons in each momentum bin for both RS and WS tracks, as described in Eq. (3). The elements of $A_{\text {PID }}$ for each dataset are determined by applying our PID requirements to MC samples of particles originating from $D_{s}^{+}$ decays. Differing detector conditions of the three datasets introduce deviations among the PID rates from the datasets. The rates that populate the $A_{\text {PID }}$ matrices are shown in Fig. 5 for each dataset. The results of the unfolding procedure for both RS and WS tracks are shown in Fig. 6, as well as the difference of the PIDunfolded right-sign and wrong-sign yields, which gives the PID-unfolded momentum spectra for tracked positrons originating from $D_{s}^{+} \rightarrow X e^{+} \nu_{e}$ and $D_{s}^{+} \rightarrow \tau^{+} \nu_{\tau} \rightarrow$ $e^{+} \nu_{e} \nu_{\tau} \bar{\nu}_{\tau}$ events. The assessment of systematic uncertainties related to the two rates to which we are most sensitive (the electron identification efficiency and the pion-to-electron misidentification rate) are discussed in Sec. VI.
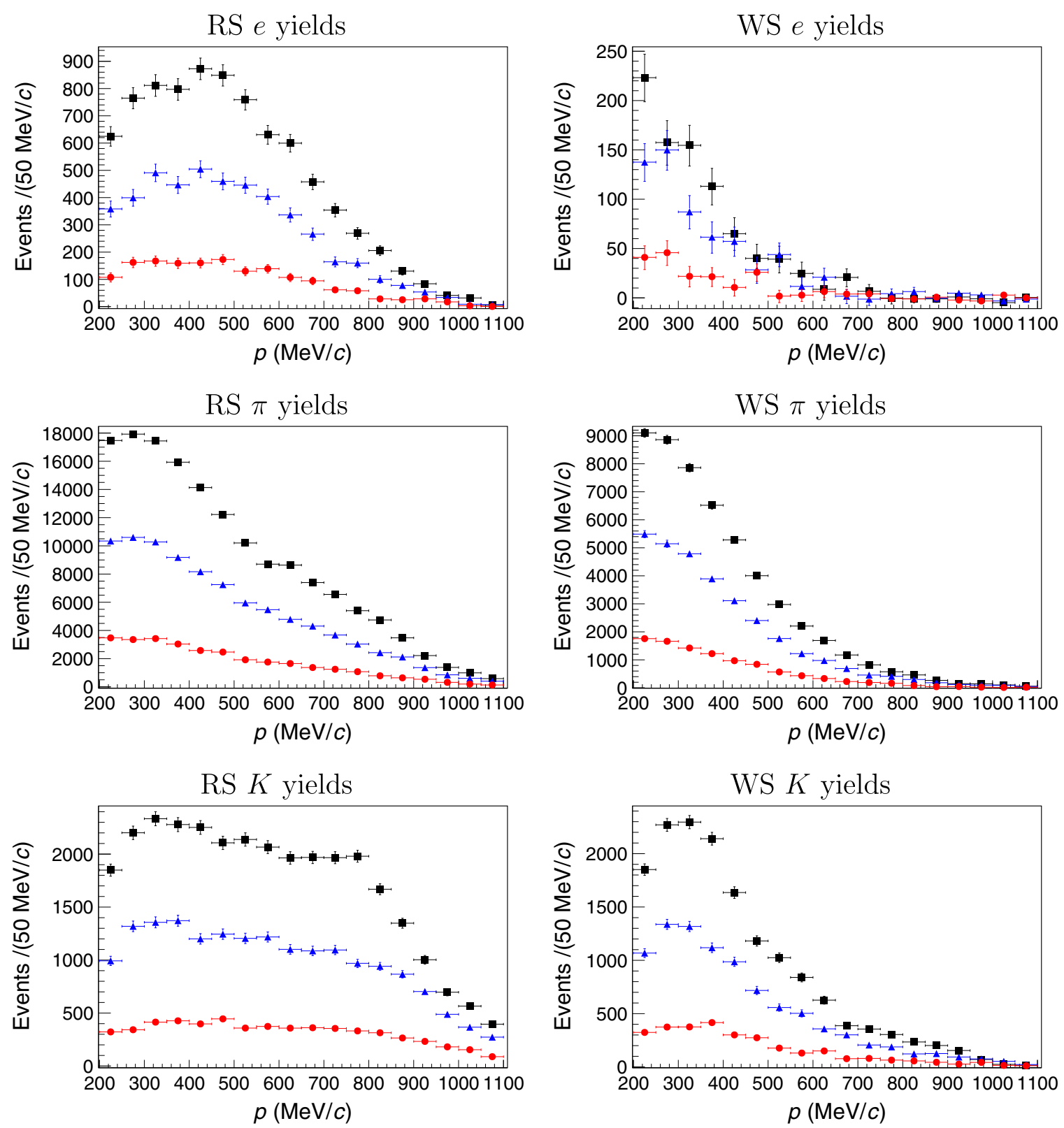

FIG. 4. Measured yields for each track category as a function of momentum. The results from $E_{\mathrm{cm}}=4.178 \mathrm{GeV}$ are shown as black squares, those from $E_{\mathrm{cm}}=4.189-4.219 \mathrm{GeV}$ are shown as blue triangles, and those from $E_{\mathrm{cm}}=4.225-4.230 \mathrm{GeV}$ are shown as red circles. 

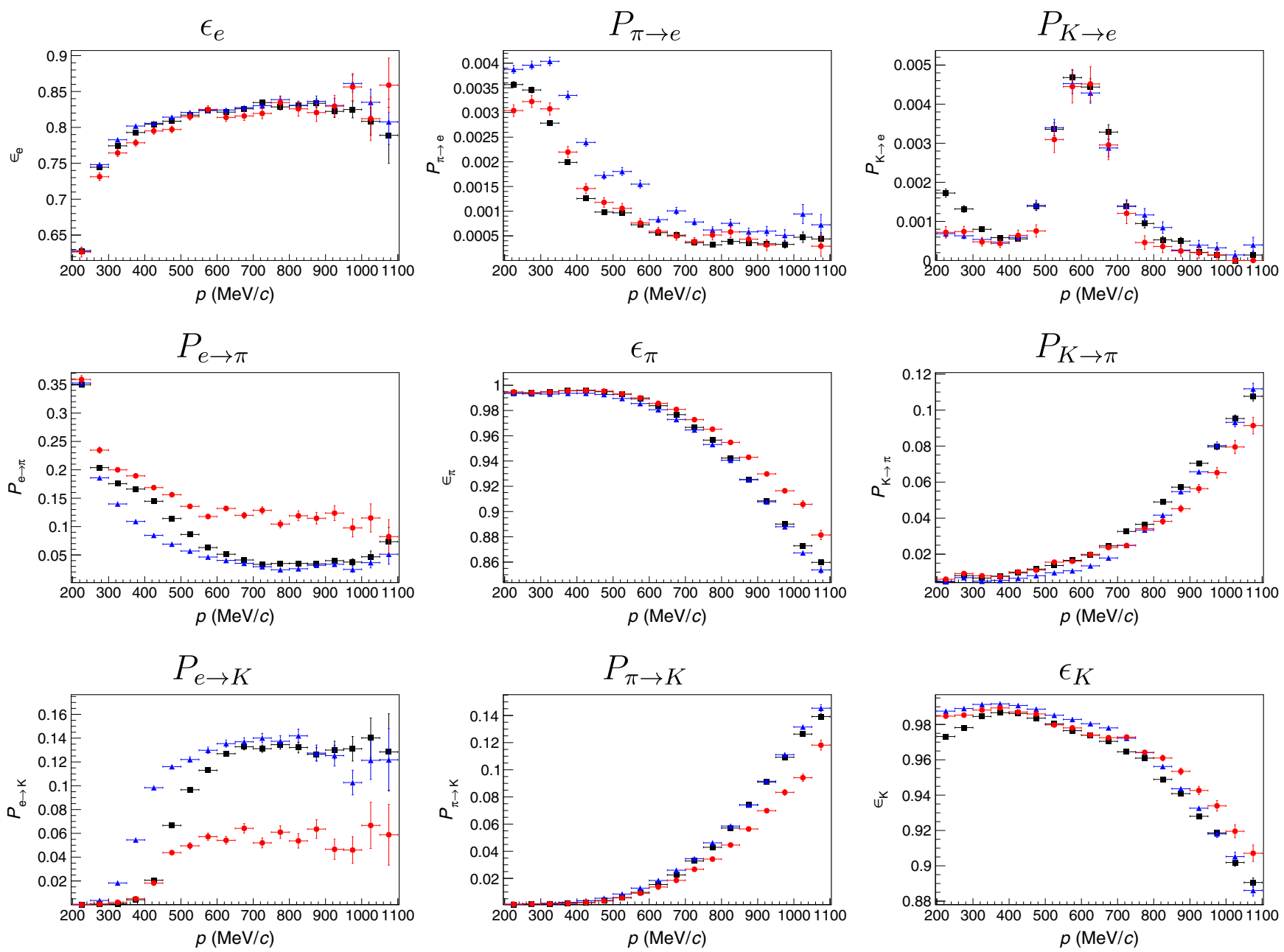

FIG. 5. PID rates as a function of momentum used to populate the $A_{\mathrm{PID}}$ matrices. Entries from $E_{\mathrm{cm}}=4.178 \mathrm{GeV}$ are shown as black squares, those from $E_{\mathrm{cm}}=4.189-4.219 \mathrm{GeV}$ are shown as blue triangles, and those from $E_{\mathrm{cm}}=4.225-4.230 \mathrm{GeV}$ are shown as red circles.

After taking the difference of the RS and WS PIDcorrected yields, we apply the tracking unfolding matrix to correct for tracking reconstruction efficiencies and momentum bin misassignment as in Eq. (4). The momentum bin misassignment is caused not only by imperfect detector resolution but also by FSR from electrons and positrons, which increases the likelihood for the track momentum to be less than the momentum produced in the $D_{s}^{+}$decay. The tracking unfolding matrices are consistent among the MC samples.

We determine the $D_{s}^{+} \rightarrow X e^{+} \nu_{e}$ positron momentum spectrum for each data sample by subtracting the contribution of positrons from $D_{s}^{+} \rightarrow \tau^{+} \nu_{\tau} \rightarrow e^{+} \nu_{e} \nu_{\tau} \bar{\nu}_{\tau}$ events from the spectra obtained from the tracking unfolding procedure. We take the $D_{s}^{+} \rightarrow \tau^{+} \nu_{\tau} \rightarrow e^{+} \nu_{e} \nu_{\tau} \bar{\nu}_{\tau}$ positron momentum spectra from MC samples. We fix the normalization according to

$$
N_{\mathrm{DT}, \tau}=\mathcal{B}\left(D_{s}^{+} \rightarrow \tau^{+} \nu_{\tau} \rightarrow e^{+} \nu_{e} \nu_{\tau} \bar{\nu}_{\tau}\right) \times n_{\mathrm{ST}} \times b_{\mathrm{tag}}^{\tau},
$$

where the values of $\mathcal{B}\left(D_{s}^{+} \rightarrow \tau^{+} \nu_{\tau} \rightarrow e^{+} \nu_{e} \nu_{\tau} \bar{\nu}_{\tau}\right)$ and $b_{\text {tag }}^{\tau}$ are given in Tables I and IV, respectively. The subtracted $\tau$ component is shown for each dataset in Fig. 7.

By determining the signal-efficiency-corrected number of $D_{s}^{+} \rightarrow X e^{+} \nu_{e}$ events in each dataset with $p_{e}>200 \mathrm{MeV} / c$, all effects of the detector response, except for small effects in $b_{\text {tag }}$, have been accounted for. As such, we sum the determined yields from each dataset in each momentum bin to produce a combined $D_{s}^{+} \rightarrow X e^{+} \nu_{e}$ momentum spectrum. A table with the total summed yields as a function of momentum along with their statistical uncertainties is provided in the Supplemental Material [36]. We determine the number of $D_{s}^{+} \rightarrow X e^{+} \nu_{e}$ events with $p_{e} \leq 200 \mathrm{MeV} / c$ by fitting a shape based on MC simulation to the combined yields with $p_{e}>200 \mathrm{MeV} / c$. The shape is constructed by adding the momentum spectra predicted by $\mathrm{MC}$ simulation of the six observed exclusive modes $\left(\phi e^{+} \nu_{e}, \eta e^{+} \nu_{e}, \eta^{\prime} e^{+} \nu_{e}, K^{0} e^{+} \nu_{e}, K^{*}(892)^{0} e^{+} \nu_{e}\right.$, and $\left.f_{0}(980) e^{+} \nu_{e}\right)$ in proportion to their branching fractions 

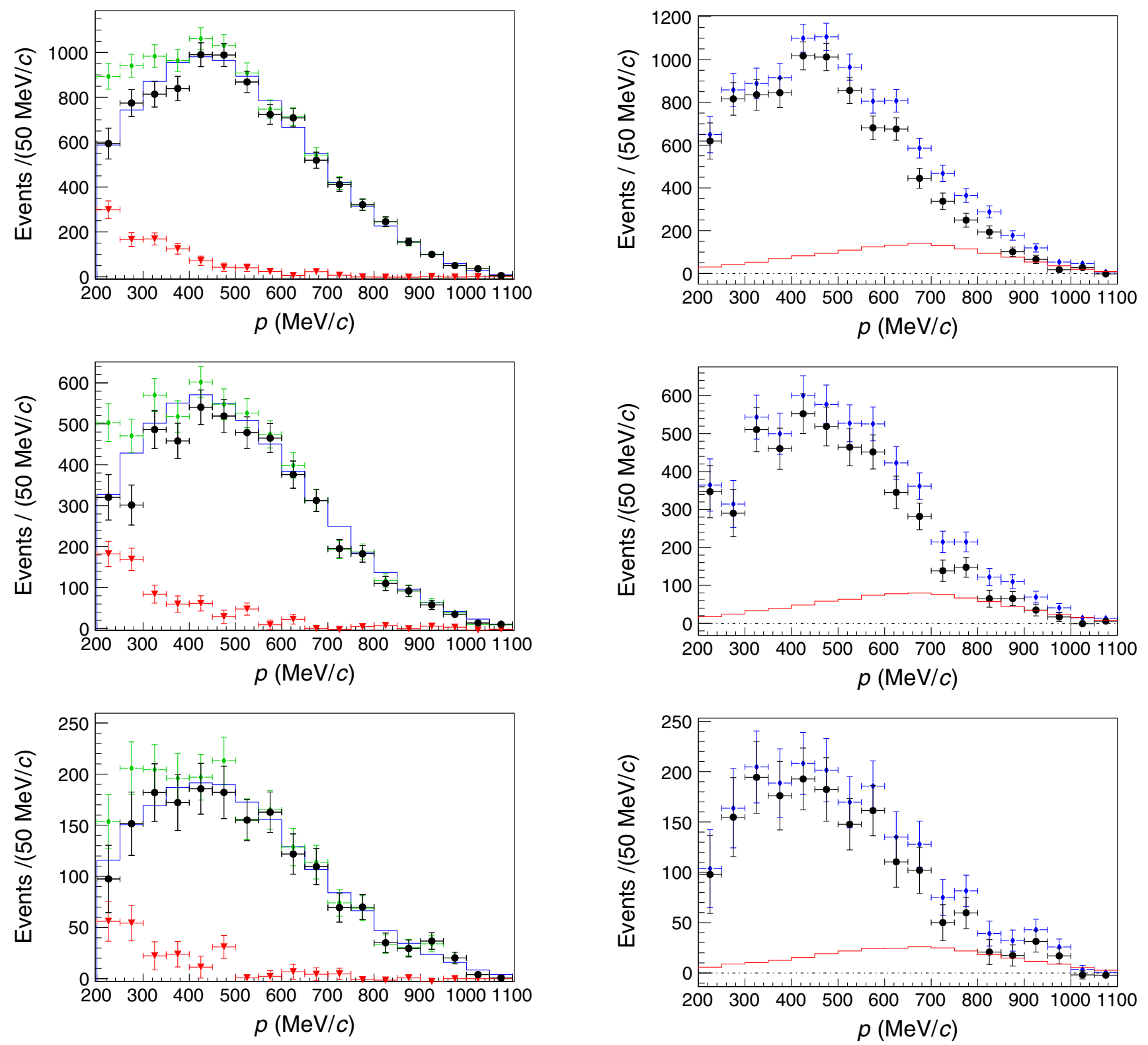

FIG. 6. PID unfolding results of positrons from $D_{s}^{+} \rightarrow X e^{+} \nu_{e}$ and $D_{s}^{+} \rightarrow \tau^{+} \nu_{\tau} \rightarrow e^{+} \nu_{e} \nu_{\tau} \bar{\nu}_{\tau}$ events for the datasets with $E_{\mathrm{cm}}=$ $4.178 \mathrm{GeV}$ (upper), $E_{\mathrm{cm}}=4.189-4.219 \mathrm{GeV}$ (middle), and $E_{\mathrm{cm}}=4.225-4.230 \mathrm{GeV}$ (lower). RS PID-corrected yields are shown as green diamonds, WS PID-corrected yields are shown as red triangles, their differences are shown as black circles, and the predictions from MC samples scaled by the number of single-tag events are shown as blue histograms.

listed in Table I. The momentum spectra for the $\eta^{\prime} e^{+} \nu$, $K^{0} e^{+} \nu_{e}, K^{*}(892)^{0} e^{+} \nu_{e}$, and $f_{0}(980) e^{+} \nu_{e}$ modes are taken from MC samples generated with the ISGW2 model [37] for the decay of the $D_{s}^{+}$meson. We generate separate MC samples for the two largest modes, $\phi e^{+} \nu_{e}$ and $\eta e^{+} \nu_{e}$, using simple-pole parametrizations of the respective decay form factors as functions of the 4-momentum squared of the lepton-neutrino system $\left(q^{2}\right)$. The form-factor parameters

FIG. 7. Results of subtracting the contribution of $D_{s}^{+} \rightarrow$ $\tau^{+} \nu_{\tau} \rightarrow e^{+} \nu_{e} \bar{\nu}_{\tau} \nu_{\tau}$ in $E_{\mathrm{cm}}=4.178 \mathrm{GeV}$ data (upper), $E_{\mathrm{cm}}=$ 4.189-4.219 GeV data (middle), and $E_{\mathrm{cm}}=4.225-4.230 \mathrm{GeV}$ data (lower). Tracking-corrected yields are shown as blue diamonds, and the predicted contributions from $D_{s}^{+} \rightarrow \tau^{+} \nu_{\tau} \rightarrow$ $e^{+} \nu_{e} \nu_{\tau} \bar{\nu}_{\tau}$ are shown as red histograms. The differences of the two, the $D_{s}^{+} \rightarrow X e^{+} \nu_{e}$ yields, are shown as black circles. The dotted black line indicates zero events.

are taken from measurements of the $B A B A R$ Collaboration for $\phi e^{+} \nu_{e}$ [3] and the BESIII Collaboration for $\eta e^{+} \nu_{e}$ [7].

The integral of the fitted spectrum from $p_{e}=0 \mathrm{MeV} / c$ to $p_{e}=200 \mathrm{MeV} / c$ is added to the number of signalefficiency-corrected $D_{s}^{+} \rightarrow X e^{+} \nu_{e}$ events with $p_{e}>$ $200 \mathrm{MeV} / c$ as described in Eq. (5), and the statistical uncertainty is scaled by the ratio by which the total yield increases due to this correction. The fit to data with the 


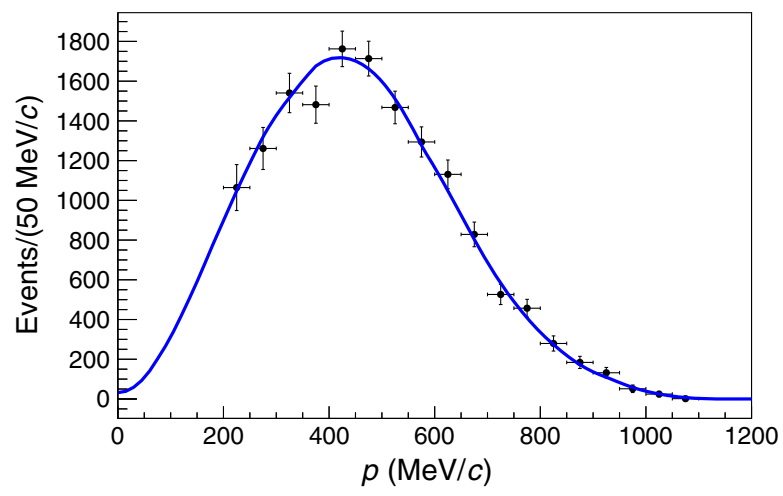

FIG. 8. Momentum spectrum fit used to determine the $D_{s}^{+}$ semielectronic decay yield below $200 \mathrm{MeV} / c$. The black points with error bars are sums of the final measured $D_{s}^{+} \rightarrow X e^{+} \nu_{e}$ yields (black circles) for the three datasets from Fig. 7, and the solid blue line is result of the fit described in the text.

assumed momentum spectrum can be found in Fig. 8. As a cross-check, we also fit to the datasets separately and find consistent results. The yields in data both without and with the correction for the data with $p_{e}<200 \mathrm{MeV} / c$ are shown in Table V.

Using Eq. (6), we determine $\mathcal{B}\left(D_{s}^{+} \rightarrow X e^{+} \nu_{e}\right)$ with the momentum-extrapolated number of signal-efficiency-corrected double-tag events from Table $\mathrm{V}$, the observed number of single-tag events from Table III, and the tag bias from Table IV. The branching fractions determined from each dataset independently, their average, and the branching fraction determined from their combination are

TABLE V. Yields in data before and after momentum-extrapolation correction. The "Combined" row shows the results of summing together the yields from the three datasets and fitting to the summed distribution. Shown uncertainties are only statistical.

\begin{tabular}{lcc}
\hline \hline Sample & $\begin{array}{c}\text { Observed yields with } \\
p_{e}>200 \mathrm{MeV} / c\end{array}$ & $\begin{array}{c}\text { Corrected } \\
\text { yields }\left(N_{\mathrm{DT}}\right)\end{array}$ \\
\hline$E_{\mathrm{cm}}=4.178 \mathrm{GeV}$ & $8793 \pm 218$ & $9628 \pm 238$ \\
$E_{\mathrm{cm}}=4.189-4.219 \mathrm{GeV}$ & $4695 \pm 174$ & $5142 \pm 191$ \\
$E_{\mathrm{cm}}=4.225-4.230 \mathrm{GeV}$ & $1712 \pm 104$ & $1874 \pm 114$ \\
Combined & $15201 \pm 298$ & $16648 \pm 326$ \\
\hline \hline
\end{tabular}

TABLE VI. $\mathcal{B}\left(D_{s}^{+} \rightarrow X e^{+} \nu_{e}\right)$ determined from data. The "Combined" row shows the results of summing together the yields from the three datasets and fitting to the summed distribution. Shown uncertainties are only statistical.

\begin{tabular}{lc}
\hline \hline Sample & $\mathcal{B}\left(D_{s}^{+} \rightarrow X e^{+} \nu_{e}\right)$ \\
\hline$E_{\mathrm{cm}}=4.178 \mathrm{GeV}$ & $(6.38 \pm 0.16) \%$ \\
$E_{\mathrm{cm}}=4.189-4.219 \mathrm{GeV}$ & $(5.96 \pm 0.23) \%$ \\
$E_{\mathrm{cm}}=4.225-4.230 \mathrm{GeV}$ & $(6.38 \pm 0.40) \%$ \\
Combined & $(6.30 \pm 0.13) \%$ \\
\hline \hline
\end{tabular}

TABLE VII. Systematic uncertainties in the measurement of $\mathcal{B}\left(D_{s}^{+} \rightarrow X e^{+} \nu_{e}\right)$.

\begin{tabular}{lc}
\hline \hline Source & Relative uncertainty \\
\hline Tracking & $0.7 \%$ \\
PID & $0.8 \%$ \\
Spectrum extrapolation & $0.7 \%$ \\
Background shapes & $0.4 \%$ \\
Number of tags & $0.6 \%$ \\
Tag bias & $0.1 \%$ \\
$\mathcal{B}\left(D_{s}^{+} \rightarrow \tau^{+} \nu_{\tau}\right)$ & $0.6 \%$ \\
Overall & $1.6 \%$ \\
\hline \hline
\end{tabular}

shown along with the associated statistical uncertainties in Table VI. The difference of the "Combined" result from Table VI, $\mathcal{B}\left(D_{s}^{+} \rightarrow X e^{+} \nu_{e}\right)=(6.30 \pm 0.13$ (stat. $\left.)\right) \%$, and the sum of the observed exclusive semielectronic branching fractions from Table I gives the unobserved $D_{s}^{+}$semielectronic branching fraction as $(-0.04 \pm 0.21) \%$, where the stated uncertainty includes the total uncertainty from the exclusive measurements, but only the statistical uncertainty from the inclusive measurement presented in this article.

\section{SYSTEMATIC UNCERTAINTY}

Our methods to determine the relative systematic uncertainty in our measured $\mathcal{B}\left(D_{s}^{+} \rightarrow X e^{+} \nu_{e}\right)$ are described below.

\section{A. MC simulation statistics and matrix-inversion stability}

We probe the effects of finite MC sample statistics and the stability of the matrix-inversion algorithm by creating a toy ensemble of variations for each efficiency matrix used in the analysis (54 PID and three tracking matrices). For each matrix, we create this ensemble by sampling each entry within the MC sample's statistical uncertainty. For each matrix in each ensemble, we perform the matrix inversion and reperform the analysis with the new inverted matrix. All variations produce a negligible change in our final result, which indicates a negligible systematic uncertainty from the statistical uncertainty of the MC samples as well as the stability of the algorithm for inverting our efficiency matrices.

\section{B. Tracking}

Simulation of our tracking efficiency is studied with a control sample of radiative Bhabha events. Tracking efficiencies as a function of momentum are measured in each dataset as well as in MC samples produced with the BabaYaganto package [28]. The ratios of the measured efficiencies in data and MC samples are weighted by the predicted momentum distribution from signal MC simulation and the number of single-tag events in each dataset to 
determine the systematic uncertainty. This results in a relative systematic uncertainty of $0.7 \%$.

In addition, we investigate the systematic uncertainty in the individual tracking efficiency matrix entries. As we assign a systematic uncertainty for the total tracking efficiency, we probe the uncertainty in the individual entries by keeping the sum of a row of the matrix constant while varying the individual entries. The specific variation is as follows:

(i) $\left(A_{\text {trk }}\right)_{i, i-1} \Rightarrow 1.5\left(A_{\text {trk }}\right)_{i, i-1}$

(ii) $\left(A_{\text {trk }}\right)_{i, i+1} \Rightarrow 1.25\left(A_{\text {trk }}\right)_{i, i+1}$

(iii) $\left(A_{\text {trk }}\right)_{i, i}$ decreases to keep the sum of the column constant.

This variation is chosen as a conservative estimation of the uncertainties from FSR and detector resolution. We see negligible change when we perform such a variation, so we only assign the previously stated uncertainty for tracking.

\section{PID}

Similar to our procedure in assessing the systematic uncertainty in our tracking efficiencies, we measure $e$ ID efficiencies as a function of momentum and track angle in radiative Bhabha control samples for each dataset.

We also probe the accuracy of the pion-to-electron misidentification rates from MC simulation via a control sample of pions collected in each data sample through the decay chains $D^{*+} \rightarrow \pi^{+} D^{0}, D^{0} \rightarrow K^{-} \pi^{+}, K^{-} \pi^{+} \pi^{+} \pi^{-}$. To determine the total uncertainty from PID rates, we simultaneously vary the $e$ ID efficiencies and the pion-toelectron misidentification rates using the central values of the measured data-to-MC efficiency ratios and reperform our analysis. This yields a $0.8 \%$ change in the final branching fraction, which we assign as the relative systematic uncertainty due to PID.

As our sensitivity to kaon-to-electron misidentification rates is small due to the relatively few number of kaons, the systematic uncertainty in kaon-to-electron misidentification rates is neglected.

\section{Tag bias}

We follow the procedure laid out in Ref. [38], which assigns a fraction of $1-b_{\text {tag }}$ as the systematic uncertainty based on the particles in the final state of the single-tag $D_{s}^{-}$ decay. The specific guidelines for variation of detectorresponse parameters are as follows: $1.0 \%$ per kaon for tracking, $0.5 \%$ per pion for tracking, and $0.5 \%$ per kaon or pion for PID. For $D_{s}^{-} \rightarrow K^{+} K^{-} \pi^{-}$, with two kaons and one pion, the quadrature sum is $2.9 \%$. With $b_{\text {tag }}$ from Table IV (including the contribution from $D_{s}^{+} \rightarrow \tau^{+} \nu_{\tau} \rightarrow e^{+} \nu_{e} \nu_{\tau} \bar{\nu}_{\tau}$ ), taking $2.9 \%$ of $1-b_{\text {tag }}$ yields a $0.03 \%$ relative systematic uncertainty. We additionally propagate the uncertainties in the branching fractions (Table I) through the calculation of the weighted-average $b_{\text {tag }}$. This yields a $0.07 \%$ relative systematic uncertainty in the bias. We add these in quadrature and assign the relative systematic uncertainty due to tag bias as $0.1 \%$.

\section{E. Number of single tags}

We investigate the systematic uncertainty in the invariant-mass fitting procedure used to determine the number of single tags by varying the choice of background distribution from the nominal second-order Chebyshev polynomial. We use both first-order and third-order Chebyshev polynomials as variations in fitting to each dataset. Using the first-order Chebyshev polynomial gives a larger difference in the yields in all cases, while not significantly degrading the quality of the fit. We take an average of the changes for each dataset weighted by the single-tag yields to determine the systematic uncertainty, which results in a $0.6 \%$ relative systematic uncertainty in the number of single tags.

\section{F. Background shapes}

To assess the uncertainty due to our chosen background shapes in our signal-side fits of the tag invariant mass, we use smoothed background distributions from MC simulation instead of the nominal first-order Chebyshev polynomial to model backgrounds in each of the signal-side fits. We then reperform the analysis with the yields determined from these alternative fits. The relative difference in $N_{\mathrm{DT}}$ is $0.4 \%$, which we assign as the relative systematic uncertainty due to this source.

$$
\text { G. } \mathcal{B}\left(D_{s}^{+} \rightarrow \boldsymbol{\tau}^{+} \boldsymbol{\nu}_{\boldsymbol{\tau}}\right)
$$

As systematic uncertainty in the kinematic distributions of $D_{s}^{+} \rightarrow \tau^{+} \nu_{\tau} \rightarrow e^{+} \nu_{e} \nu_{\tau} \bar{\nu}_{\tau}$ events contributes negligible uncertainty, we account for uncertainty in the contribution of $D_{s}^{+} \rightarrow \tau^{+} \nu_{\tau} \rightarrow e^{+} \nu_{e} \nu_{\tau} \bar{\nu}_{\tau}$ events by propagating the absolute uncertainty for $\mathcal{B}\left(D_{s}^{+} \rightarrow \tau^{+} \nu_{\tau} \rightarrow e^{+} \nu_{e} \nu_{\tau} \bar{\nu}_{\tau}\right)$ from Table I, $0.04 \%$. This yields a relative systematic uncertainty of $0.6 \%$ on our measurement of $\mathcal{B}\left(D_{s}^{+} \rightarrow X e^{+} \nu_{e}\right)$.

\section{H. Spectrum extrapolation}

We assess the uncertainty due to the momentum spectrum extrapolation by generating an ensemble of alternative momentum spectra and fitting these to the data. Each spectrum in this ensemble is created by Gaussian sampling the branching fractions of the six observed exclusive semielectronic modes and adding spectra for unobserved decay modes. We then add these spectra in proportion based on the sampled branching fractions.

We consider effects from the combination of three unobserved decay modes: $D_{s}^{+} \rightarrow h_{1}(1415) e^{+} \nu_{e}, D_{s}^{+} \rightarrow$ $f_{1}(1510) e^{+} \nu_{e}$, and $D_{s}^{+} \rightarrow \gamma e^{+} \nu_{e}$. MC samples for $D_{s}^{+} \rightarrow$ $h_{1}(1415) e^{+} \nu_{e}$ and $D_{s}^{+} \rightarrow f_{1}(1510) e^{+} \nu_{e}$ are generated based on the ISGW2 model's predictions [37]. We generate MC samples for $D_{s}^{+} \rightarrow \gamma e^{+} \nu_{e}$ based on the model of Yang and Yang [39]. We determine the normalization of the 
$D_{s}^{+} \rightarrow h_{1}(1415) e^{+} \nu_{e}$ and $D_{s}^{+} \rightarrow f_{1}(1510) e^{+} \nu_{e}$ spectra by fixing the relative branching fraction of these decays to the ISGW2 predictions and fitting them in addition to our nominal momentum spectrum. The $D_{s}^{+} \rightarrow \gamma e^{+} \nu_{e}$ spectrum is fixed to its measured $90 \%$ confidence-level upper limit, $1.3 \times 10^{-4}$ [40]. From this fit, we determine an upper limit at the $90 \%$ confidence level for the sum of the branching fractions of $D_{s}^{+} \rightarrow h_{1}(1415) e^{+} \nu_{e}$ and $D_{s}^{+} \rightarrow$ $f_{1}(1510) e^{+} \nu_{e}$. In our toy ensemble, their summed spectra are fixed based on this upper limit. We performed this same procedure excluding the $D_{s}^{+} \rightarrow \gamma e^{+} \nu_{e}$ spectrum but found the combination of modes to produce the largest variation.

The resulting systematic uncertainty is determined by filling a distribution of the relative change in $\mathcal{B}\left(D_{s}^{+} \rightarrow\right.$ $\left.X e^{+} \nu_{e}\right)$ between the alternative momentum spectra and our nominal spectrum. The linear sum of the mean $(0.33 \%)$ and rms $(0.34 \%)$ of this distribution is taken as the uncertainty, which gives a $0.7 \%$ relative systematic uncertainty.

We also probe uncertainty in the models we employ by using the ISGW2-predicted momentum spectra for the $D_{s}^{+} \rightarrow \phi e^{+} \nu_{e}$ and $D_{s}^{+} \rightarrow \eta e^{+} \nu_{e}$ modes. Using these alternative spectra instead of the nominal spectra gives a less than $0.1 \%$ difference in the measured branching fraction, so we conclude that any uncertainty due to model dependence in our analysis is negligible.

\section{Summary of systematic uncertainties}

The assigned relative systematic uncertainties for all sources are listed in Table VII. Our systematic uncertainty is not dominated by any single source, but the largest contributions come from the momentum spectrum extrapolation and imperfect simulation of PID and tracking efficiencies. The total relative systematic uncertainty is obtained from the quadrature sum of the assigned relative uncertainties. This gives a total relative systematic uncertainty of $1.6 \%$.

\section{CONCLUSIONS}

Using data collected by the BESIII detector in the centerof-mass-energy range of $E_{\mathrm{cm}}=4.178-4.230 \mathrm{GeV}$, we measure the inclusive semielectronic branching fraction of the $D_{s}^{+}$meson to be

$$
\begin{aligned}
& \mathcal{B}\left(D_{s}^{+} \rightarrow X e^{+} \nu_{e}\right) \\
& \quad=(6.30 \pm 0.13 \text { (stat. }) \pm 0.09(\text { syst. }) \pm 0.04(\text { ext. })) \%
\end{aligned}
$$

where the external systematic uncertainty is from $\mathcal{B}\left(D_{s}^{+} \rightarrow \tau^{+} \nu_{\tau}\right)$. We also measure the laboratory-frame momentum spectrum of the positrons produced in this decay, which can be seen in Fig. 8.

Our result is consistent with the measurement from the CLEO-c experiment [1],

$$
\left.\left.\mathcal{B}\left(D_{s}^{+} \rightarrow X e^{+} \nu_{e}\right)=(6.52 \pm 0.39 \text { (stat. }) \pm 0.15 \text { (syst. }\right)\right) \%,
$$

with a factor of 3 reduction of the statistical uncertainty and a factor of 1.5 reduction of the systematic uncertainty, primarily due to increased precision on external inputs. The total precision of our measurement is $2.6 \%$, which corresponds to approximately a 2.5 times improvement in the total precision compared to the measurement from CLEO-c.

By taking the difference between our measurement of $\mathcal{B}\left(D_{s}^{+} \rightarrow X e^{+} \nu_{e}\right)$ and the sum of the best available measurements for the exclusive semielectronic modes in Table I, we calculate the unobserved semielectronic branching fraction to be

$$
\begin{aligned}
\mathcal{B}( & \left.D_{s}^{+} \rightarrow X e^{+} \nu_{e}\right)-\sum_{i} \mathcal{B}\left(D_{s}^{+} \rightarrow X_{i} e^{+} \nu_{e}\right) \\
& =(-0.04 \pm 0.13 \text { (stat. }) \pm 0.09 \text { (syst. }) \pm 0.17 \text { (ext. })) \%,
\end{aligned}
$$

where the external systematic uncertainty includes the total uncertainty in the measured exclusive branching fractions and the uncertainty in $\mathcal{B}\left(D_{s}^{+} \rightarrow \tau^{+} \nu_{\tau}\right)$. Our measurement provides no evidence for the existence of unobserved $D_{s}^{+}$ semileptonic decay modes and constrains the branching fractions of all unobserved decay modes. In addition, the measured momentum spectrum can be used to further constrain the decay rates of modes with characteristic momentum spectra. The spectrum is included in tabular form in the Supplemental Material [36].

With our updated measurement of the $D_{s}^{+}$semielectronic branching fraction, the CLEO-c measurement of the $D^{0} 0$ semielectronic branching fraction [1], and the 2020 PDG values for the $D_{s}^{+}$and $D^{0} 0$ lifetimes [35], we find

$$
\begin{aligned}
& \frac{\Gamma\left(D_{s}^{+} \rightarrow X e^{+} \nu_{e}\right)}{\Gamma\left(D^{0} \rightarrow X e^{+} \nu_{e}\right)} \\
& \quad=0.790 \pm 0.016 \text { (stat. }) \pm 0.011 \text { (syst.) } \pm 0.016 \text { (ext.) }
\end{aligned}
$$

where the external systematic uncertainty includes the total uncertainty from $\mathcal{B}\left(D^{0} 0 \rightarrow X e^{+} \nu_{e}\right)$, the $D^{0}$ and $D_{s}^{+}$lifetimes, and $\mathcal{B}\left(D_{s}^{+} \rightarrow \tau^{+} \nu_{\tau}\right)$. This result is in agreement with the prediction of $\frac{\Gamma\left(D_{s}^{+} \rightarrow X e^{+} \nu\right)}{\Gamma\left(D^{0} 0 \rightarrow X e^{+} \nu\right)}=0.813$ from Ref. [18], supporting the conclusion that the difference in the semileptonic decay widths of $D_{s}^{+}$and $D^{0} 0$ mesons can be accounted for within the Standard Model by nonspectator interactions. Further theoretical analysis of our measured spectrum, similar to those of Refs. [19,20], can constrain specific processes like weak annihilation of the constituent $c$ and $\bar{s}$ quarks of the $D_{s}^{+}$meson, with potential extensions to determinations of $\left|V_{u b}\right|$ in semileptonic $B$ decays [21].

\section{ACKNOWLEDGMENTS}

The BESIII Collaboration thanks the staff of BEPCII and the IHEP computing center for their strong support. This work is supported in part by National Key Research and 
Development Program of China under Contracts No. 2020YFA0406400 and No. 2020YFA0406300; National Natural Science Foundation of China (NSFC) under Contracts No. 11625523, No. 11635010, No. 11735014, No. 11822506, No. 11835012, No. 11935015, No. 11935016, No. 11935018, and No. 11961141012; the Chinese Academy of Sciences (CAS) Large-Scale Scientific Facility Program; Joint Large-Scale Scientific Facility Funds of the NSFC and CAS under Contracts No. U1732263 and No. U1832207; CAS Key Research Program of Frontier Sciences under Contract No. QYZDJ-SSW-SLH040; 100 Talents Program of CAS; INPAC and Shanghai Key Laboratory for Particle Physics and Cosmology; ERC under Contract No. 758462; European Union Horizon 2020 research and innovation programme under Marie Sklodowska-Curie Grant No. 894790; German Research Foundation DFG under Contract No. 443159800 and Collaborative Research Center Grants No. CRC 1044, No. FOR 2359, No. FOR 2359, and No. GRK 214; Istituto Nazionale di Fisica Nucleare, Italy; Ministry of Development of Turkey under Contract No. DPT2006K-120470; National Science and Technology fund; Olle Engkvist Foundation under Contract No. 200-0605; STFC (United Kingdom); The Knut and Alice Wallenberg Foundation (Sweden) under Contract No. 2016.0157; The Royal Society, UK under Contracts No. DH140054 and No. DH160214; The Swedish Research Council; and U.S. Department of Energy under Contracts No. DE-FG02-05ER41374 and No. DE-SC-0012069.
[1] D. M. Asner et al. (CLEO Collaboration), Phys. Rev. D 81, 052007 (2010).

[2] K. M. Ecklund et al. (CLEO Collaboration), Phys. Rev. D 80, 052009 (2009).

[3] B. Aubert et al. (BABAR Collaboration), Phys. Rev. D 78, 051101 (2008).

[4] L. Zhang (BESIII Collaboration), BEAUTY 2019, Results about Charm Decays at BESIII, https://indico.ijs.si/event/ 1031/contributions/145/attachments/103/113/bes3charm decays_beauty2019_LeiZhang.pdf.

[5] M. Ablikim et al. (BESIII Collaboration), Phys. Rev. D 97, 012006 (2018).

[6] J. Hietala, D. Cronin-Hennessy, T. Pedlar, and I. Shipsey, Phys. Rev. D 92, 012009 (2015).

[7] M. Ablikim et al. (BESIII Collaboration), Phys. Rev. Lett. 122, 121801 (2019).

[8] M. Ablikim et al. (BESIII Collaboration), Phys. Rev. D 94, 112003 (2016).

[9] M. Ablikim et al. (BESIII Collaboration), Phys. Rev. Lett. 122, 061801 (2019).

[10] M. Ablikim et al. (BESIII Collaboration), Phys. Rev. D 94, 072004 (2016).

[11] A. Zupanc et al. (Belle Collaboration), J. High Energy Phys. 09 (2013) 139.

[12] P. del Amo Sanchez et al. (BABAR Collaboration), Phys. Rev. D 82, 091103 (2010).

[13] J. Alexander et al. (CLEO Collaboration), Phys. Rev. D 79, 052001 (2009).

[14] P. Naik et al. (CLEO Collaboration), Phys. Rev. D 80, 112004 (2009).

[15] P. U. E. Onyisi et al. (CLEO Collaboration), Phys. Rev. D 79, 052002 (2009).

[16] I. I. Bigi and N. G. Uraltsev, Z. Phys. C 62, 623 (1994).

[17] M. B. Voloshin, Phys. Lett. B 515, 74 (2001).

[18] M. Gronau and J. L. Rosner, Phys. Rev. D 83, 034025 (2011).
[19] Z. Ligeti, M. Luke, and A. V. Manohar, Phys. Rev. D 82, 033003 (2010).

[20] P. Gambino and J. F. Kamenik, Nucl. Phys. B840, 424 (2010).

[21] M. Fael, T. Mannel, and K. K. Vos, J. High Energy Phys. 12 (2019) 067.

[22] C. Yu et al., in Proceedings, 7th International Particle Accelerator Conference (IPAC 2016), Busan, Korea (CERN, Geneva, 2016).

[23] M. M. Ma et al., Radiat. Detect. Technol. Methods 3, 58 (2019).

[24] M. Ablikim et al. (BESIII Collaboration), Nucl. Instrum. Methods Phys. Res., Sect. A 614, 345 (2010).

[25] S. Jadach, B. F. L. Ward, and Z. Was, Comput. Phys. Commun. 130, 260 (2000); Phys. Rev. D 63, 113009 (2001).

[26] D. J. Lange, Nucl. Instrum. Methods Phys. Res., Sect. A 462, 152 (2001).

[27] R. G. Ping, Chin. Phys. C 32, 599 (2008).

[28] C. M. Carloni Calame, H. Czyż, J. Gluza, M. Gunia, G. Montagna, O. Nicrosini, F. Piccinini, T. Riemann, and M. Worek, Nucl. Phys. B225-B227, 293497 (2012).

[29] E. Richter-Was, Phys. Lett. B 303, 163 (1993).

[30] S. Agostinelli et al., Nucl. Instrum. Methods Phys. Res., Sect. A 506, 250 (2003).

[31] Y. T. Liang, B. Zhu, Z. Y. You, K. Liu, and H. X. Ye, Nucl. Instrum. Methods Phys. Res., Sect. A 603, 325 (2009).

[32] M. Ablikim et al. (BESIII Collaboration), Chin. Phys. C 40, 063001 (2016).

[33] M. Ablikim et al. (BESIII Collaboration), Chin. Phys. C 39, 093001 (2015).

[34] R. M. Baltrusaitis et al. (MARK III Collaboration), Phys. Rev. Lett. 56, 2140 (1986); J. Adler et al. (MARK III Collaboration), Phys. Rev. Lett. 60, 89 (1988).

[35] A. Zyla et al. (Particle Data Group), Prog. Theor. Exp. Phys. 2020, 083C01 (2020) 
[36] See Supplemental Material at http://link.aps.org/ supplemental/10.1103/PhysRevD.104.012003 which includes a table of signal efficiency-corrected yields and the full set of fits used in determining the double-tag yields.

[37] D. Scora and N. Isgur, Phys. Rev. D 52, 2783 (1995).
[38] M. Ablikim et al. (BESIII Collaboration), Phys. Rev. D 99, 031101(R) (2019).

[39] J. C. Yang and M. Z. Yang, Nucl. Phys. B889, 778 (2014).

[40] M. Ablikim et al. (BESIII Collaboration), Phys. Rev. D 99, 072002 (2019). 